\title{
An Exact Connection between two Solvable SDEs and a Nonlinear Utility Stochastic PDE *†
}

\author{
El Karoui Nicole, ${ }^{\ddagger} \quad$ Mrad Mohamed $\S$
}

November 30, 2012

\begin{abstract}
Motivated by the work of Musiela and Zariphopoulou [24], we study the Itô random fields which are utility functions $U(t, x)$ for any $(\omega, t)$. The main tool is the marginal utility $U_{x}(t, x)$ and its inverse expressed as the opposite of the derivative of the Fenchel conjuguate $\widetilde{U}(t, y)$. Under regularity assumptions, we associate a $\operatorname{SDE}(\mu, \sigma)$ and its adjoint $\operatorname{SPDE}(\mu, \sigma)$ in divergence form whose $U_{x}(t, x)$ and its inverse $-\widetilde{U}_{y}(t, y)$ are monotonic solutions. More generally, special attention is paid to rigorous justification of the dynamics of inverse flow of SDE. So that, we are able to extend to the solution of similar SPDEs the decomposition based on the solutions of two SDEs and their inverses.

The second part is concerned with forward utilities, consistent with a given incomplete financial market, that can be observed but given exogenously to the investor. As in 24], market dynamics are considered in an equilibrium state, so that the investor becomes indifferent to any action she can take in such a market. After having made explicit the constraints induced on the local characteristics of consistent utility and its conjugate, we focus on the marginal utility SPDE by showing that it belongs to the previous family of SPDEs. The associated two SDE's are related to the optimal wealth and the optimal state price density, given a pathwise explicit representation of the marginal utility. This new approach addresses several issues with a new perspective: dynamic programming principle, risk tolerance properties, inverse problems. Some examples and applications are given in the last section
\end{abstract}

\section{Introduction}

The concept of forward dynamic utility has been introduced by M. Musiela and T. Zariphopoulou (2003-2008 [24, 25, 27, 29]), to model possible changes over the time of individual preferences

\footnotetext{
*With the financial support of the "Chaire Risque Financier of the Fondation du Risque", the " Chaire Dérivés du futur" of the "Fédération des banques Françaises".

${ }^{\dagger}$ Keywords: Forward utility, performance criteria, horizon-unbiased utility, consistent utility, progressive utility, portfolio optimization, optimal portfolio, duality, minimal martingale measure, stochastic flows, stochastic partial differential equations.

${ }^{\ddagger}$ LPMA, UMR CNRS 6632, Université Pierre et Marie Curie, CMAP, UMR CNRS 7641, École Polytechnique

§CMAP, UMR CNRS 7641, École Polytechnique, LAGA, UMR CNRS 7539, Université Paris 13
} 
of an agent. The economic agent will adjust its preferences in uncertain universe based on the information that is revealed over time and represented by a filtration $\mathbb{F}=\left(\mathcal{F}_{t}\right)_{t \geq 0}$, defined on the probability space $(\Omega, \mathbb{P}, \mathcal{A})$. In contrast to the classical literature, there is no pre-specified trading horizon at the end of which the utility datum is assigned. Rather, the agent starts with today's specification of its utility, $U(0, x)=u(x)$, and then builds the process $U(t, x)$ for $t>0$ in relation to the information given by $\left(\mathcal{F}_{t}\right)_{t \geq 0}$. This, together with the choice of an initial utility, distinguishes forward dynamic utility from recursive utility first introduced by Duffie and Epstein, [4], (see also [7]) for which the aggregator can be specified exogenously and the value function is recovered backward in time. Dynamic utility may be also defined in relation with a exogenous investment universe $\mathcal{X}$, described as an incomplete market whose the parameters are known by the investor. Following [24], market dynamics are considered in an equilibrium state, so that the investor becomes indifferent to any action she can take in such a market. She is forced to embed into his dynamic utility, structure information describing the equilibrium market dynamics. Such $\mathcal{X}$ - consistent utilities will be studied in the second part of the paper.

Such concept has also been studied by Berrier, Tehranchi and Rogers (2009) 1 and Zitkovic [41]. Further works related to this problem are Choulli, Stricker and Jia (2007) [36], Henderson and Hobson (2007) [11].

We start by introducing the general notion of progressive utility and its Fenchel transform. However, we restrict our study to positive progressive utility which is a collection of Itô's semimartingales with respect to a $d$-dimensional Brownian motion $W$, depending on a spatial parameter $x \in \mathbb{R}^{+}$, also called the wealth. As usual, they are specified through their local (drift, volatility) characteristics $(\beta, \gamma)$ and their initial condition, a deterministic concave function $u$, by

$$
d U(t, x)=\beta(t, x) d t+\gamma(t, x) . d W_{t}, \quad U(0, x)=u(x) .
$$

Apart regularity issues, the first question to be solved is to give conditions on the local characteristics $(\beta, \gamma)$, insuring the properties of monotonicity and concavity of the progressive utility $\mathbf{U}$ defined by the forward equation (1.1). The problem is equivalent to show that the progressive marginal utility $\mathbf{U}_{x}$ is strictly decreasing and strictly positive, with range $(0, \infty)$. At this stage, it is convenient to introduce the Fenchel conjugate random field $\widetilde{\mathbf{U}}$ of $\mathbf{U}$ whose the opposite of the derivative $\widetilde{\mathbf{U}}_{\mathbf{y}}$ is the inverse flow of $\mathbf{U}_{x}$. Following Kunita [19], we introduce sufficient conditions implying that $\mathbf{U}_{x}$ is also an Itô progressive random field, with local characteristics $\left(\beta_{x}, \gamma_{x}\right)$. (Theorem 2.2 All regularity issues are presented in details in Section 2.)

Then we show that the random field $\mathbf{U}_{x}$ is a monotonic with respect to its initial condition, strong solution of a one dimensional stochastic differential equation. The random coefficients of the $\operatorname{SDE}(\mu, \sigma)$ are expressed in terms of the local characteristics $\beta_{x}$ and $\gamma_{x}$, and $-\widetilde{U}_{y}$. We give in particular, under some Lipschitz assumptions on SDE's coefficients, a clear answer to the existence, monotonicity, regularity and concavity of random fields defined from (0.1) satisfying the Inada's conditions, which until now remained an open question.

Additional regularity are need to show that the conjugate random field $\widetilde{U}$ and its derivative $\widetilde{U}_{y}$ are also families of Itô's semimartingales, and to identify its local characteristics. The Itô-Ventzel formula enables us to carry out computations in a stochastically modulated dynamic framework. Applied to the random field $\mathbf{U}$ and the semimartingale $\widetilde{U}_{y}(t, y)$, Itô-Ventzel's formula provides us 
two complementary results: the dynamics of $\widetilde{U}$, and the characterization of $\widetilde{U}_{y}(t, y)$ as monotonic solution of the SPDE generated by the adjoint elliptic operator in divergence form associated with the $\operatorname{SDE}(\mu, \sigma)$ satisfied by $\mathbf{U}_{x}$. A first connection between SDE and SPDE is then established. Section 2 is more technical. Based on the reference book of Kunita 19], we provide additional results in both regularity of random fields, and behavior of SDE with local Lipschitz property. The aim is to provide conditions under which the inverse of a monotonic solution of $\operatorname{SDE}(\mu, \sigma)$ (for instance the process $\left.-\widetilde{U}_{y}(t, y)\right)$ is an Itô semimartingale, solution of the second-order $\operatorname{SPDE}(\mu, \sigma)$ (Theorem 2.5). Conversely, a regular monotonic solution of $\operatorname{SPDE}(\mu, \sigma)$ is identified with the inverse of the monotonic solution of $\operatorname{SDE}(\mu, \sigma)$. The link between $\operatorname{SDE}$ and second order $\operatorname{SPDE}$ is extended to more complex SPDE in the main result of this section, Theorem 2.7 .

In the second part of the paper, we turn to consistent stochastic utilities, where the preferences are no more defined in isolation with a financial market. The dynamics of the market can be observed but are exogenously given to the investor, modulo estimation of the relevant parameters by the equilibrium considerations or other procedures. Following [24], market dynamics are considered in an equilibrium state, so that the investor becomes indifferent to any action she can take in such a market. But, once characterized, the criterion may be used to solve classical optimization problems as optimal allocation, indifference pricing [33].

Working with positive wealth processes $X^{\kappa}$ in an incomplete market, we define a consistent stochastic utility as a progressive non-negative stochastic utility $U(t, x)$, for which $U\left(t, X_{t}^{\kappa}\right)$ is a supermartingale, and a (local) martingale for a certain portfolio called optimal wealth, or optimal benchmark. Section 3 is dedicated to set-up the financial market, and to identify the constraints induced on the local characteristics $(\beta, \gamma)$ of $\mathbf{U}$ by the consistency property, using once again the Itô-Ventzel formula. As in the classical Hamilton-Jacobi-Bellman framework, we proceed by verification to establish the dynamics of consistent utilities. Assuming a sufficient constraint on the drift $\beta$ of HJB type, we get the utility SPDE that we investigate in this paper. In particular, we study the role of the utility risk premium defined by $\eta^{U}(t, x)=\gamma_{x}(t, x) / U_{x}(t, x)$. Simultaneously, we go into details on the duality questions and give a characterization of the nonlinear SPDE satisfied by the progressive convex conjugate $\widetilde{U}$ of $U$, and its optimal coefficients.

Our most original contribution is provided in Section 4 using a characterization of $\mathcal{X}$-consistent dynamic utility from its marginal utility. Based on ideas developed in Section 2, we show that, under regularity assumptions on the random field $\mathbf{U}, U_{x}$ is solution of a SPDE(2.9) associated with the optimal coefficients for the wealth and state price density processes. Then, a first result shows that under natural assumptions yielding to the existence of strong optimal solution of the conjugate problem $Y_{t}^{*}(y)$, the only locally Lipschitz $\operatorname{SDE}\left(\mu^{*}, \sigma^{*}\right)$ has a monotonic non-explosive solution $X_{t}^{*}(x)$. A simple application of Theorem 2.7 yields to the closed form of the marginal utility as $U_{x}(t, x)=Y_{t}^{*}\left(u_{x}\left(\left(X_{t}^{*}\right)^{-1}(x)\right)\right)$.

Then, we consider the converse problem of recovering a dynamic utility coherent with a given optimal portfolio. In the classical case, this problem is known in the financial literature as the "inverse" Merton problem; it has been considered by many authors in particular by H.He and C.Huang (1992), [10]. Since the class of dynamic utilities is larger than the class of Markovian utilities considered in [10], our problem is easier to solve. Given a monotonic admissible portfolio $\mathbf{X}$, for any regular state price density process $Y_{t}(y)$ and any initial condition $u_{x}$ such that $V(t, x)=Y_{t}^{*}\left(u_{x}\left(\left(X_{t}\right)^{-1}(x)\right)\right)$ is integrable near to 0, is the marginal utility of some consistent dy- 
namic utility. So, we are able to generate all the consistent utilities with a given optimal portfolio. In Section 4. Theorem 4.1 we establish the most original contribution of this paper. Indeed, from Theorem 2.7, focusing on the dynamics of the marginal utility $U_{x}$, we can read directly and without difficulties that the process $U_{x}\left(t, X_{t}^{*}(x)\right)$ is equal to the optimal dual state price density process $Y^{*}\left(t, u_{x}(x)\right)$ with $u$ is the initial utility function. From this dual identity, the idea is very simple and natural: Suppose that the optimal portfolio denoted by $\left(X_{t}^{*}(x)\right)_{t}$ is strictly increasing with respect to the initial capital, and denote by $(\mathcal{X}(t, x))_{t}$ the adapted inverse process, defined by $X_{t}^{*}(\mathcal{X}(t, x))=x$ then, we can find $U_{x}(t, x)$ from $U_{x}(t, x)=Y_{t}^{*}\left(u_{x}(\mathcal{X}(t, x))\right)$. Finally we get $U$ by integration. So, we are able to generate all the consistent utilities with a given optimal portfolio. We recover easily the martingale property of the risk tolerance established in [10], in a complete market. Finally, in Section 5, we close the paper by some openness to other topics and works; we show the stability of the notion of consistent utility by change of numeraire and then, without loss of generality, we can consider the martingale market where the portfolios are simple local martingales and the stochastic PDE's are easier to deal with. We also apply our method to the specific example of decreasing consistent utilities (see [1] and [28]) where the volatility vector $\gamma$ is given equal to zero, given a new interpretation of the optimal wealth as solution of sup-convolution problems in random power utilities.

To the best of our knowledge, the characterization of strictly concave increasing random fields, satisfying Inada's conditions, from SDEs have neither studied in details nor established in the literature. Moreover, the fully nonlinear utility stochastic PDE's established in this paper and satisfied by forward utilities and their dual have not been established in a general way. Furthermore, there is no general consistent utilities construction proposed in the literature, expect the case of power or exponential type, or decreasing utilities. Another main contribution of this paper is a connection between two solvable SDEs and the utility SPDE early established. In particular, given a volatility vector $\gamma$ such that $\gamma_{x}(t, x)=-x U_{x x} \kappa_{t}^{*}(x)-U_{x}(t, x) \eta_{t}^{\mathcal{R}}+U_{x}(t, x) \nu_{t}^{*}\left(U_{x}(t, x)\right)$ (where $\eta^{\mathcal{R}}$ is the market risk prime), we show the existence and uniqueness of a solution to the fully nonlinear second order SPDE from that of a pair of SDE's. In any case this, represents an interesting result in the theory of stochastic partial differential equations.

The paper is organized as follows, in the next section we give the definition of the Itô progressive dynamic utilities and focus on the characterization of these concave Itô's random fields by establishing a link with SDEs. A special attention is paid to the dynamics of the Fenchel conjuguate utility random field. In the following section, we give sufficient conditions ensuring concavity, monotonicity, differentiability both for random fields or for solution of SDE. The dynamics of the inverse of regular solution of $\operatorname{SDE}(\mu, \sigma)$ is the first step in the links of $\operatorname{SDE}$ and SPDE. More complex situations are then studied.

Next, we introduce the consistent utilities, the market model and the framework of the paper. In Section 3, we provide, simultaneously, the dynamics of consistent utilities and their convex conjugate. We establish a closed form for the optimal policies. Thereafter, in Section 4 we establish a fully characterization of the marginal utility. We end by reverse engineering problem and some openness to other topics. 


\section{Progressive Utility}

In a dynamic and stochastic environment, the classical notion of utility is not flexible enough to help us to make good choices in the long run. M.Musiela and T.Zariphopoulou were the first to suggest to use instead of the classical criterion the concept of progressive dynamic utility, consistent with respect to a given investment universe in a sense specified in Section 3. The concept of progressive utility gives an adaptative way to include new information on environment evolution available to economic agents. Recently, Fritelli [8] introduced very closed notion called stochastic dynamic utility, in view of study certainty equivalent. Since these utility functions are stochastic, time dependent and moving forward, we consider them as a family of (Itô) semimartingales depending on a parameter, the wealth of the agent in the economic context.

\subsection{Definition and Properties of Progressive Dynamic Utility}

All stochastic processes are defined on a standard filtered probability space $(\Omega, \mathbb{F}, \mathbb{P})$, where the filtration $\mathbb{F}=\left(\mathcal{F}_{t}\right)_{t \geq 0}$ is assumed to be right continuous and complete. We first recall some notions relative to stochastic processes depending of a parameter.

Generality on progressive random fields Stochastic processes in consideration are depending on a parameter, often called spatial parameter. For us, because of economic motivation, this parameter is the wealth of an investor, taking non negative values in $\mathbb{R}_{+}^{*}=\{x>0\}$. Sometimes, we will use the vocabulary of random field theory, and refer to such processes as progressive random fields. As all random fields considered in the sequel are progressive, we will often omit the mention " progressive".

(i) A progressive random field $\mathbf{X}=\{X(t, x) ; t \geq 0, x>0\}$ is a random variable measurable with respect to $\mathcal{F}_{\infty} \otimes \mathcal{B}\left(\mathbb{R}_{+}\right) \otimes \mathcal{B}\left(\mathbb{R}_{+}^{*}\right)$, which is a collection of progressive processes $t \mapsto X(t, x)$.

(ii) A random field $\mathbf{X}$ is said to satisfy a property $\mathcal{P}$ with respect to $x$, if there exists $N \in \mathcal{F}_{\infty}$ with $\mathbb{P}(N)=0$, such that for any $\omega \in N^{c}$, and any $t \geq 0, x \mapsto X(t, x)(\omega)$ satisfies the property $\mathcal{P}$. For example, $\mathbf{X}$ is said to be concave, (resp. increasing) if there exists $N \in \mathcal{F}_{\infty}$ with $\mathbb{P}(N)=0$, such that for any $\omega \in N^{c}$, and any $t \geq 0 x \mapsto X(t, x)(\omega)$ is concave (resp. increasing).

(iii) A random field $\mathbf{X}$ is said to be continuous if there exists $N \in \mathcal{F}_{\infty}$ with $\mathbb{P}(N)=0$, such that for any $\omega \in N^{c}$, and any $t \geq 0 x \mapsto X(t, x)(\omega)$ is continuous. In general, the existence of continuous modification is based of the Kolmogorov's criterion, that we recall in Section 2

A random field $\mathbf{X}$ is said to be differentiable if there exists $N \in \mathcal{F}_{\infty}$ with $\mathbb{P}(N)=0$, such that for any $\omega \in N^{c}$, and any $t \geq 0 x \mapsto X(t, x)(\omega)$ is differentiable ; the derivative denoted $X_{x}(t, x)(\omega)$ generates the so-called derivative random field $\mathbf{X}_{x}$. When $\mathbf{X}_{x}$ has a continuous version, $\mathbf{X}$ is said to be $\mathcal{C}^{1}$-regular.

(iv) A d-dimensional random field $\mathbf{X}$ is said to be $\mathcal{L}^{k}$ - locally bounded if for any compact $\left.K \subset\right] 0, \infty[$, there exists $N \in \mathcal{F}_{\infty}$ with $\mathbb{P}(N)=0$, such that for any $\omega \in N^{c}, \int_{0}^{T} \sup _{x \in K}\|X(t, x)\|^{k}(\omega) d t<\infty$ for any $T>0$.

In the sequel, we are concerned with differentiable random fields said to be $\mathcal{K}_{\text {loc }}^{m}$-regular $(\mathcal{K}$ for Kunita) in the following sense. 
Definition 1.1. A d-dimensional random field $\mathbf{X}$ is said to be $\mathcal{K}_{\text {loc }}^{m}$ (resp. $\overline{\mathcal{K}}_{\text {loc }}^{m}$ )-regular if $X$ is $a \mathcal{C}^{m}$-regular random field such that $X / x, \partial_{x}^{k} X, k \leq m$ are $\mathcal{L}^{1}$ (resp. $\mathcal{L}^{2}$ )-locally bounded. Such random fields are also called of class $\mathcal{K}_{\text {loc }}^{m}\left(\right.$ resp. $\left.\overline{\mathcal{K}}_{\text {loc }}^{m}\right)$.

At this stage, we content ourselves with this definition to carry out calculations in this section; more details are given in the next section.

Progressive utility and its Fenchel conjugate We start with the definition of a progressive utility as progressive random field with concavity property.

Definition 1.2 (Progressive Utility). A progressive utility is a continuous progressive random field on $\mathbb{R}_{+}^{*}, \mathbf{U}=\{U(t, x) ; t \geq 0, x>0\}$ such that,

(i) UTILITY PROPERTY: U is strictly concave, strictly increasing, and non negative.

(ii) Regularity PROPERTy: $\mathbf{U}$ is a $\mathcal{C}^{2}$-random field, with continuous first and second derivatives random fields $\mathbf{U}_{\mathbf{x}}$ and $\mathbf{U}_{\mathbf{x x}}$.

(iii) InAda CONDitions: $\mathbf{U}$ goes to 0 when $x$ goes to 0 and the derivative $\mathbf{U}_{\mathbf{x}}$ goes to $\infty$ when $x$ goes to 0 , and to 0 when $x$ goes to $\infty$.

Given its importance in convex analysis, we introduce together with any progressive utility U, its convex conjugate $\widetilde{\mathbf{U}}$ (also called conjugate progressive utility (CPU)), that is the Fenchel Legendre transform of the convex random field $-\mathbf{U}(,-$.).

Definition 1.3 (Progressive conjugate utility). The convex conjugate of the progressive utility $\mathbf{U}$ is the progressive random field $\widetilde{\mathbf{U}}$ defined on $\mathbb{R}_{+}^{*}$ by $\widetilde{\mathbf{U}}=\{\widetilde{U}(t, y) ; t \geq 0, y>0\}$, where $\widetilde{U}(t, y) \stackrel{\text { def }}{=}$ $\max _{x>0, x \in Q^{+}}(U(t, x)-x y)$.

(i) Under Inada condition, $\widetilde{\mathbf{U}}$ is twice continuously differentiable, strictly convex, strictly decreasing, with $\widetilde{U}\left(., 0^{+}\right)=U(+\infty), \widetilde{U}(.,+\infty)=U\left(0^{+}\right)$, a.s.

(ii) The marginal utility $\mathbf{U}_{x}$ is the inverse of the opposite of the marginal conjugate utility $\widetilde{\mathbf{U}}_{y}$, that is $U_{x}(t, .)^{-1}(y)=-\widetilde{U}_{y}(t, y)$, with Inada conditions $\widetilde{U}_{y}\left(., 0^{+}\right)=-\infty, \widetilde{U}_{y}(.,+\infty)=0$

(iii) The bi-dual relation holds true $U(t, x)=\inf _{y>0, y \in Q^{+}}(\widetilde{U}(t, y)+x y)$. Moreover $\widetilde{U}(t, y)=U(t,-\widetilde{U}(t, y))+\widetilde{U}_{y}(t, y) y$, and $U(t, x)=\widetilde{U}\left(t, U_{x}(t, x)\right)+x U_{x}(t, x)$.

\subsection{Itô Progressive Utility and SDE}

In this paper, we focus on continuous progressive utilities $\mathbf{U}$ which are a collection of Itô semimartingales: for any $x, U(., x)$ is a continuous Itô semimartingale, driven by a $n$-dimensional Brownian motion $W=\left(W^{1}, . ., W^{n}\right)$ defined on the probability space $(\Omega, \mathbb{F}, \mathbb{P})$. In general, the $\sigma$ field $\mathcal{F}_{0}$ is assumed to be trivial and $\mathcal{F}_{0^{-}}$random variables are a.s. constant. We refer to the book of H.Kunita [19] and to the next section for all technical results concerning the theory of semimartingale random fields. The assumption of finite dimensional Brownian motion greatly simplifies the theory.

As usual, an Itô random field $\mathbf{F}$ defined on $\mathbb{R}^{+}$is specified through its decomposition $\mathbf{F}=$ $F_{0}+\mathbf{B}^{F}+\mathbf{M}^{F}$ into two random fields, where $\mathbf{B}^{F}$ is a finite variation random field $B^{F}(t, x)=$ $\int_{0}^{t} \varphi^{F}(s, x) d s$ and $\mathbf{M}^{F}$ is a martingale random field $M^{F}(t, x)=\int_{0}^{t} \psi^{F}(s, x) d W_{s}$, where $\left(\varphi^{F}, \psi^{F}\right)=$ $\left\{\left(\varphi^{F}(t, x), \psi^{F}(t, x)\right) ; t \geq 0, x>0\right\}$ are the local characteristics of $F$ assumed to be progressive random fields, with values in $\mathbb{R}$ and $\mathbb{R}^{n}$ respectively. $\varphi^{F}$ is called the drift characteristic, and $\psi^{F}$ the 
diffusion characteristic. For simplicity, we often omit the index $F$. By convention, an Itô random field $\mathbf{F}$ is said to be a $\mathcal{K}_{l o c}^{m}$-semimartingale, whenever $\mathbf{B}^{F}$ is of class $\mathcal{K}_{l o c}^{m}$, and $\mathbf{M}^{F}$ is of class $\overline{\mathcal{K}}_{l o c}^{m}$. The reference to $\mathcal{K}^{m}$ recall that $\mathbf{F}$ is a random field.

Let us consider an Itô progressive utility $U$ with initial condition $u(x)=U(0, x)$, and local characteristics $(\beta, \gamma)$, so that

$$
d U(t, x)=\beta(t, x) d t+\gamma(t, x) \cdot d W_{t}, \quad U(0, x)=u(x)
$$

Apart regularity issues, the first question before going further is the following:

How to express by conditions on the local characteristics $(\beta, \gamma)$ random fields, the properties of monotonicity and concavity of the progressive utility $\mathbf{U}$ defined by (1.1)?

The problem is equivalent to show that the progressive marginal utility $\mathbf{U}_{x}$ is strictly decreasing and strictly positive, with range $(0, \infty)$. Following Kunita [19], this can be done by assuming that, (i) $U$ is of class $\mathcal{K}_{\text {loc }}^{2}$, which implies in particular (Theorem 2.2 below) that $\mathbf{U}_{x}$ is also an Itô progressive random field, with local characteristics $\left(\beta_{x}, \gamma_{x}\right)$.

(ii) The random field $\mathbf{U}_{x}$ is a strong solution of a one dimensional stochastic differential equation (SDE), with random coefficient, monotonic with respect to its initial condition.

We summarize these ideas in the following theorem.

Theorem 1.1. Let $\mathbf{U}$ be an Itô random field with dynamics

$$
d U(t, x)=\beta(t, x) d t+\gamma(t, x) \cdot d W_{t}, \quad U(0, x)=u(x)
$$

(i) NeCESSARY CONDITION Let $\mathbf{U}$ be a progressive utility with conjugate utility $\widetilde{\mathbf{U}}$, and marginal utilities $U_{x}(t,$.$) and -\widetilde{U}_{y}(t,$.$) . If \mathbf{U}$ is a $\mathcal{K}_{l o c}^{2}$-semimartingale, the random field $Z .(z)=U_{x}\left(.,-\tilde{u}_{y}(z)\right)$ is a strictly increasing (in $z$ ) solution of the $S D E(\mu, \sigma)$,

$$
\left\{\begin{aligned}
d Z_{t} & =\mu\left(t, Z_{t}\right) d t+\sigma\left(t, Z_{t}\right) d W_{t}, & & Z_{0}=z \\
\mu(t, z) & =\beta_{x}\left(t,-\widetilde{U}_{y}(t, z)\right), & & \mu(t, 0)=0 \\
\sigma(t, z) & :=\gamma_{x}\left(t,-\widetilde{U}_{y}(t, z)\right), & & \sigma(t, 0)=0
\end{aligned}\right.
$$

(ii) Characterization as PRimitive of SDE Let consider a $S D E(\hat{\mu}, \hat{\sigma}), d Z_{t}=\hat{\mu}\left(t, Z_{t}\right) d t+$ $\hat{\sigma}\left(t, Z_{t}\right) d W_{t}, Z_{0}=z$ and assume the existence of a strong solution $Z .(z)$, increasing and differentiable in $z$ with range $(0, \infty)$. Then, for any utility function u such that $Z .\left(u_{x}(x)\right)$ is integrable in a neighborhood of $x=0$, the primitive $\mathbf{U}=\left\{U(t, x)=\int_{0}^{x} Z_{t}\left(u_{x}(z)\right) d z, t \geq 0, x>0\right\}$ is a progressive utility.

Proof. By assumption, from Theorem 2.2 below, $U$ is a $\mathcal{K}_{\text {loc }}^{2}$-semimartingale implies that $U_{x}$ is an Itô semimartingale with local characteristics $\left(\beta_{x}, \gamma_{x}\right)$, i.e., $d U_{x}(t, x)=\beta_{x}(t, x) d t+\gamma_{x}(t, x) . d W_{t}$. Since $x \mapsto U(t, x)$ is strictly concave and increasing, the marginal utility $U_{x}(t,$.$) is strictly positive$ and decreasing from $\infty$ to 0 . Consequently $x \mapsto U_{x}(t, x)$ has an inverse which is the opposite of the marginal conjugate utility $-\widetilde{U}_{y}(t,$.$) . By denoting \mu(t,):.=\beta_{x}\left(t,-\widetilde{U}_{y}(t,).\right)$ and $\sigma(t,):.=$ $\gamma_{x}\left(t,-\widetilde{U}_{y}(t,).\right)$, it follows that $U_{x}$ satisfies the following SDE, with initial condition $U_{x}(0, x)=$ $u_{x}(x)$

$$
d U_{x}(t, x)=\mu\left(t, U_{x}(t, x)\right) d t+\sigma\left(t, U_{x}(t, x)\right) \cdot d W_{t}
$$


The change of initial condition from $u_{x}(x)$ into $z$ yields to the definition of the process $Z_{t}(z)=$ $U_{x}\left(t,-\tilde{u}_{y}(z)\right)$. The converse implication is obvious.

It remains to give sufficient conditions on the random coefficients $(\mu, \sigma)$ ensuring the existence of a strong, monotonic solution of SDE (1.3). We briefly recall some classical results on SDEs, useful for our study; more details and additional results are provided in Section 2 An easy to read presentation of SDEs with stochastic coefficients is given in Protter [32]; for more exhaustive study, see Kunita [19].

Theorem 1.2. Let us consider the one-dimensional stochastic differential equation, $S D E(\mu, \sigma)$

$$
d Z_{t}=\mu\left(t, Z_{t}\right) d t+\sigma\left(t, Z_{t}\right) d W_{t}
$$

We assume that the $\mathbb{R}$-valued drift coefficient $\mu=\{\mu(t, x) ; t \geq 0, x \geq 0\}$ and the $\mathbb{R}^{d}$ - valued diffusion coefficent $\sigma=\{\sigma(t, x) ; t \geq 0, x \geq 0\}$ are Lipschitz random fields, with random Lipschitz bounds $C_{t}$ and $K_{t}$, such that a.s $\int_{0}^{T} C_{t} d t<+\infty$ and $\int_{0}^{T} K_{t}^{2} d t<+\infty$ for any $T$. In other words, for any $\omega$ outside of a negligible set $N$, for any $t, x, y$,

$$
\left\{\begin{aligned}
|\mu(t, x, \omega)-\mu(t, y, \omega)| \leq C_{t}(\omega)|x-y|, & \mu(t, 0) & \equiv 0 \\
\|\sigma(t, x, \omega)-\sigma(t, y, \omega)\| \leq K_{t}(\omega)|x-y|, & \sigma(t, 0) & \equiv 0
\end{aligned}\right.
$$

(i) Then, for any $z \in \mathbb{R}_{+}$there exists a unique strong solution $\mathbf{Z}^{z}$, also called global solution, of the $S D E(\mu, \sigma)$ (1.4) $\left(Z_{0}^{z}=z\right)$. Moreover, almost surely, the family of maps $z \mapsto Z_{t}^{z}(\omega), t \geq 0$ is continuous and strictly increasing.

(ii) The range of the map $z \mapsto Z(., z)$ is $] 0,+\infty[$ and $Z(., z)$ is integrable near to 0 and to $\infty$. More precisely,

a) When $z$ goes to $\infty$, almost surely, for any $\varepsilon \in(0,1)$, uniformly on $[0, T]$,

$$
\lim _{z \rightarrow+\infty}\left(\sup _{0 \leq t \leq T} \frac{Z(t, z)}{z^{1+\varepsilon}}\right)=0 \text { and } \lim _{z \rightarrow+\infty}\left(\sup _{0 \leq t \leq T} \frac{Z(t, z)}{z^{\varepsilon}}\right)=+\infty, \text { for any } T
$$

b) When $z$ goes to 0 , for any $\varepsilon \in(0,1)$,

$$
\lim _{z \rightarrow 0}\left(\sup _{0 \leq t \leq T} \frac{Z(t, z)}{z^{\varepsilon}}\right)=0 \text { and } \lim _{z \rightarrow 0}\left(\sup _{0 \leq t \leq T} \frac{Z(t, z)}{z^{1+\varepsilon}}\right)=+\infty, \text { for all } T
$$

Comment: (i) A constant Lipschitz bound $C$ corresponds to the classical framework of Lipschitz SDE, and the assertion (i) is well-known.

(ii) The asymptotic behavior (ii) a) (near to infinity) is less known except in the domain of stochastic flows, where several works and improvements are dealing with this behavior near of infinity, but unlike Kunita [19] and Salah-Eldin \& al [23], Imkeller \& al [13] and Zongxia [21] consider only the case of SDEs with deterministic coefficients .

(iii) The notion of "global solution" expresses that the solution $\left(Z_{t}^{z}\right)$ exists for all $t \geq 0$. Under weaker assumptions, the solution may be defined only up to a finite lifetime $\zeta(z)$. More details will be given in the next section.

Sufficient conditions on local characteristics of an Itô random field to be a progressive utility may be exhibited. 
Corollary 1.3. Assume the framework of Theorem 1.1.

(i) If there exist random Lipschitz bounds $C_{t}$ and $K_{t}$ with $\int_{0}^{T} C_{t} d t<+\infty$ and $\int_{0}^{T} K_{t}^{2} d t<+\infty$ for any $T$, such that a.s, for any $x, x^{\prime}>0$

$$
\begin{cases}\left|\beta_{x}(t, x)-\beta_{x}\left(t, x^{\prime}\right)\right| \leq C_{t}\left|U_{x}(t, x)-U_{x}\left(t, x^{\prime}\right)\right|, & \lim _{x \rightarrow \infty} \beta_{x}(t, x) \equiv 0 \\ \left|\gamma_{x}(t, x)-\gamma_{x}\left(t, x^{\prime}\right)\right| \leq K_{t}\left|U_{x}(t, x)-U_{x}\left(t, x^{\prime}\right)\right|, & \lim _{x \rightarrow \infty} \gamma_{x}(t, x) \equiv 0\end{cases}
$$

then $\mathbf{U}$ is a progressive utility.

(ii) Moreover if $(\beta, \gamma)$ are $\mathcal{C}^{2}$-regular random fields, Condition (1.8) is equivalent to

$$
\left\{\begin{aligned}
\mid \beta_{x}(t, x) & \leq C_{t}\left|U_{x}(t, x)\right|, & & \left\|\gamma_{x}(t, x)\right\| \leq K_{t}\left|U_{x}(t, x)\right| \\
\left|\beta_{x x}(t, x)\right| & \leq C_{t}^{1}\left|U_{x x}(t, x)\right|, & & \left\|\gamma_{x x}(t, x)\right\| \leq K_{t}^{1}\left|U_{x x}(t, x)\right|
\end{aligned}\right.
$$

Then $\mu(t, x)$ and $\sigma(t, x)$ have linear growth with random bounds $C_{t}$ and $K_{t}$ respectively, and $\mu_{x}$ and $\sigma_{x}$ are spatially bounded by $C_{t}^{1}$ and $K_{t}^{1}$

Proof. The condition (1.8) is equivalent to the Lipschitz condition in Theorem 1.2 applied to the coefficients $\mu$ and $\sigma$ in Equation (1.3).

Remark: In the following, we call stochastic flow any continuous and strictly monotonic solution $\mathbf{Z}$ of SDEs. Contrary to the classical theory, herein we are interested only in the process $Z_{t} \stackrel{\text { def }}{=} Z_{s=0, t}$ starting at time 0 , and its inverse $\left(Z_{t}\right)^{-1}(y)=Z_{s=0, t}^{-1}(y):=Z_{t, s=0}$, both considered in the forward point of view. We don't use the general flow associated with the SDE, defined as the family $Z_{s, t}(z)$, solution of the equation starting from $z$ at time $s$, and the flow stability, that is for $r<s<t$, $Z_{s, t}\left(Z_{r, s}(z)\right)=Z_{r, t}(z)$, except in the paragraph concerning a pathwise dynamic programming principle $(\mathrm{p} \lcm{32})$.

From Theorem 1.1, a large part of our study returns to study strictly monotonic one dimensional random fields and their inverses. The inverse flow of $\mathbf{U}_{x},\left(-\widetilde{\mathbf{U}}_{\mathbf{y}}\right)$ plays also a major role in the study of the conjugate $\widetilde{\mathbf{U}}$ of a progressive utility $\mathbf{U}$.

\subsection{Dynamics of Convex Conjugate Progressive Utility}

The study of the convex conjugate $\widetilde{\mathbf{U}}$ of a progressive utility $\mathbf{U}$ is based on the well-known identity (Definition 1.3) $\widetilde{U}(t, y)=U\left(t,-\widetilde{U}_{y}(t, y)\right)+y \widetilde{U}_{y}(t, y)$, and request to know the dynamics of the $\mathcal{C}^{2}$-random field $U(t, x)$ along the random process $-\widetilde{U}_{y}(t, y)$. For this, an extension of the classical Itô's formula, known as Itô-Ventzel's formula is needed. We refer to Ventzel [37] and Kunita [19] (Theorem 3.3.1) for different variants of this formula and theirs proofs.

Theorem 1.4 (Itô-Ventzel's Formula Weak). Consider a $\mathcal{K}_{\text {loc }}^{2}$-Itô semimartingale $\mathbf{F}$ with local characteristics $(\phi, \psi)$. For any continuous Itô semimartingale $X, F(., X$.) is a continuous Itô semimartingale,

$$
\begin{gathered}
F\left(t, X_{t}\right)=F\left(0, X_{0}\right)+\int_{0}^{t} \phi\left(s, X_{s}\right) d s+\int_{0}^{t} \psi\left(s, X_{s}\right) \cdot d W_{s} \\
+\int_{0}^{t} F_{x}\left(s, X_{s}\right) d X_{s}+\frac{1}{2} \int_{0}^{t} F_{x x}\left(s, X_{s}\right)\left\langle d X_{s}\right\rangle+\left.\int_{0}^{t}\left\langle d F_{x}(s, x), d X_{s}\right\rangle\right|_{x=X_{s}}
\end{gathered}
$$


Comment The first line of the right hand side of the equation corresponds to the dynamics of the process $(F(t, x))_{t \geq 0}$ taken on $\left(X_{t}\right)_{t \geq 0}$, when in the second line, the first two terms come from the classical Itô's formula. The last term represents the quadratic covariation between $d F_{x}(t, x)$ and $d X_{t}$, at $x=X_{t}$, which can be written as $\psi_{x}\left(t, X_{t}\right) \cdot \sigma_{t}^{X} d t$ when the diffusion coefficient of $X$ is the vector $\sigma_{t}^{X}$.

Itô-Ventzel's formula and monotonic change of variable will help us to establish the relationship between local characteristics of $\mathbf{U}$ and $\overline{\mathbf{U}}$.

Theorem 1.5. Let $\mathbf{U}$ a progressive utility and $\widetilde{\mathbf{U}}$ its progressive convex conjugate utility assumed to be $\mathcal{K}_{\text {loc }}^{2}$-Itô semimartingales with local characteristics $(\beta, \gamma)$ and $(\tilde{\beta}, \tilde{\gamma})$.

(i) The dynamics of $\widetilde{\mathbf{U}}$ is driven by the non linear second order SPDE,

$$
d \widetilde{U}(t, y)=\gamma\left(t,-\widetilde{U}_{y}(t, y)\right) \cdot d W_{t}+\beta\left(t,-\widetilde{U}_{y}(t, y)\right) d t+\frac{1}{2} \widetilde{U}_{y y}(t, y)\left\|\gamma_{x}\left(t,-\widetilde{U}_{y}(t, y)\right)\right\|^{2} d t
$$

(ii) The local characteristics of the random field $\widetilde{\mathbf{U}}_{\mathbf{y}}$ are given by,

$$
\left\{\begin{array}{l}
\tilde{\gamma}_{y}(t, y)=-\gamma_{x}\left(t,-\widetilde{U}_{y}(t, y)\right) \widetilde{U}_{y y}(t, y) \\
\tilde{\beta}_{y}(t, y)=-\beta_{x}\left(t,-\widetilde{U}_{y}(t, y)\right) \widetilde{U}_{y y}(t, y)+\frac{1}{2} \partial_{y}\left(\frac{\left\|\tilde{\gamma}_{y}(t, y)\right\|^{2}}{\widetilde{U}_{y y}(t, y)}\right)
\end{array}\right.
$$

(iii) Let $(\mu, \sigma)$ be the random coefficients of the SDE associated with $\mathbf{U}_{\mathbf{x}}$ and $\widehat{L}^{\sigma, \mu}(f)(t, y)=$ $\frac{1}{2} \partial_{y}\left(\|\sigma(t, y)\|^{2} \partial_{y} f(t, y)\right)-\mu(t, y) \partial_{y} f(t, y)$ the adjoint elliptic operator in divergence form associated with $(\mu, \sigma)$. Then the marginal conjugate utility $\widetilde{\mathbf{U}}_{\mathbf{y}}$ is a monotonic solution of the Stochastic Partial Differential Equation with initial condition $\tilde{u}_{y}(y)$,

$$
d \widetilde{U}_{y}(t, y)=-\partial_{y}\left(\widetilde{U}_{y}\right)(t, y) \sigma(t, y) \cdot d W_{t}+\widehat{L}_{t, y}^{\sigma, \mu}\left(\widetilde{U}_{y}\right) d t
$$

Observe that the derivability of the local characteristics $(\tilde{\beta}, \tilde{\gamma})$ of $\widetilde{\mathbf{U}}$ requires the existence of a third derivative for $\mathbf{U}$.

Proof. At first, the assumption " $U$ and $\widetilde{U}$ are $\mathcal{K}_{l o c}^{2}$-semimartingales" implies, from Kunita [19] Theorem 2.2 below, that $(\beta, \gamma)$ and $(\tilde{\beta}, \tilde{\gamma})$ belong to the class $\in \mathcal{K}_{l o c}^{1} \times \tilde{\mathcal{K}}_{l o c}^{1}$. Moreover $U_{x}$ and $\widetilde{U}_{y}$ are Itô semimartingales with local characteristics $\left(\beta_{x}, \gamma_{x}\right)$ and $(\tilde{\beta}, \tilde{\gamma})$.

Let now apply the Itô-Ventzel formula to the regular random field $F(t, x)=U(t, x)-y x$ and to the semimartingale $X_{t}=-\widetilde{U}_{y}(t, y)$. The following identities related to the change of variable will be useful, $F\left(t,-\widetilde{U}_{y}(t, y)\right)=\widetilde{U}(t, y), U_{x x}\left(t,-\widetilde{U}_{y}(t, y)\right)=-1 / \widetilde{U}_{y y}(t, y)$.

(i) a) Observe that $F_{x}\left(t,-\widetilde{U}_{y}(t, y)\right)=U_{x}\left(-\widetilde{U}_{y}(t, y)\right)-y \equiv 0$, so that the term in $d X$ disappears. Therefore, by Itô-Ventzel's formula, the volatility random field $\tilde{\gamma}$ of $\widetilde{\mathbf{U}}$ is $\tilde{\gamma}(t, y)=\gamma\left(t,-\widetilde{U}_{y}(t, y)\right)$, and its derivative $\tilde{\gamma}_{y}(t, y)=-\gamma_{x}\left(t,-\widetilde{U}_{y}(t, y)\right) \widetilde{U}_{y y}(t, y)$ is by assumption the volatility characteristic of $\widetilde{\mathbf{U}}_{\mathbf{y}}$. Hence the covariation term is driven by $\left\langle d F_{x}(t, x),-d \widetilde{U}_{y}(t, y)\right\rangle=-\left\langle\gamma_{x}(t, x) \cdot \tilde{\gamma}_{y}(t, y)\right\rangle d t$.

(i) b) The Itô-Ventzel formula is then reduced to,

$$
\begin{aligned}
d \widetilde{U}(t, y)- & \beta\left(t,-\widetilde{U}_{y}(t, y)\right) d t-\gamma\left(t,-\widetilde{U}_{y}(t, y)\right) \cdot d W_{t} \\
& =\frac{1}{2} U_{x x}\left(t,-\widetilde{U}_{y}(t, y)\right)\left\langle d \widetilde{U}_{y}(t, y)\right\rangle-\left\langle\gamma_{x}\left(t,-\widetilde{U}_{y}(t, y)\right) \cdot \tilde{\gamma}_{y}(t, y)\right\rangle d t \\
& =\frac{1}{2} U_{x x}\left(t,-\widetilde{U}_{y}(t, y)\right)\left\|\tilde{\gamma}_{y}(t, y)\right\|^{2} d t-U_{x x}\left(t,-\widetilde{U}_{y}(t, y)\right)\left\|\tilde{\gamma}_{y}(t, y)\right\|^{2} d t \\
& =-\frac{1}{2} U_{x x}\left(t,-\widetilde{U}_{y}(t, y)\right)\left\|\tilde{\gamma}_{y}(t, y)\right\|^{2} d t=\frac{1}{2} \widetilde{U}_{y y}(t, y)\left\|\gamma_{x}\left(t,-\widetilde{U}_{y}(t, y)\right)\right\|^{2}
\end{aligned}
$$


(ii) The dynamics of $\widetilde{\mathbf{U}}_{\mathbf{y}}$ is obtained (by assumption and Theorem 2.2) by differentiating term by term in the previous equation. The use of coefficients $\sigma(t, y)=\gamma_{x}\left(t,-\widetilde{U}_{y}(t, y)\right)$ and $\mu(t, y)=$ $\beta_{x}\left(t,-\widetilde{U}_{y}(t, y)\right)$ of the SDE associated with $\mathbf{U}_{\mathbf{x}}$ allows us to express $\widetilde{\mathbf{U}}_{\mathbf{y}}$ as the solution of a SPDE driven by the adjoint second order operator in $y, \widehat{L}_{t, y}^{\sigma, \mu}=\frac{1}{2} \partial_{y}\left(\|\sigma(t, y)\|^{2} \partial_{y}\right)-\mu(t, y) \partial_{y}$.

$$
\begin{aligned}
d \widetilde{U}_{y}(t, y) & =-\widetilde{U}_{y y}(t, y)\left[\mu(t, y) d t+\sigma(t, y) \cdot d W_{t}\right]+\partial_{y}\left(\frac{1}{2} \widetilde{U}_{y y}(t, y)\|\sigma(t, y)\|^{2}\right) d t \\
& =-\partial_{y} \widetilde{U}_{y}(t, y) \sigma(t, y) \cdot d W_{t}+\widehat{L}^{\sigma, \mu}\left(\widetilde{U}_{y}\right)(t, y) d t
\end{aligned}
$$

The proof is achieved.

Remark Obviously, we are interested in the properties of the $\operatorname{SDE}(\tilde{\mu}, \tilde{\sigma})$ associated with the monotonic random field $\widetilde{\mathbf{U}}_{\mathbf{y}}$ when $(\beta, \gamma)$ are $\mathcal{C}^{2}$-regular random fields. Given that $\tilde{\sigma}(t,-z)=$ $\frac{\gamma_{x}(t, z)}{U_{x x}(t, z)}$ and $\tilde{\mu}(t,-z)=\frac{1}{U_{x x}(t, z)}\left(\beta_{x}(t, z)-\frac{1}{2} \partial_{x}\left(\frac{\left\|\gamma_{x}(t, z)\right\|^{2}}{U_{x x}(t, z)}\right)\right)$, it is clear that these coefficients are not globally Lipschitz and the previous results (Theorem 1.2) cannot be applied directly. So, we report the study of this SDE in Section 2 Theorem 2.5, after introducing some additional tools.

\section{Regular Random Fields and Stochastic Differential Equa- tions}

There are several difficulties in the definition of semimartingales $F(t, x)$ depending on a parameter, as explained in the books of Kunita [19] and Carmona \& Nualart. 2], and their local characteristics $\left(\phi^{F}, \psi^{F}\right)$ (in short $(\phi, \psi)$ if there is no possible confusion).

(i) The first one is relative to the existence of continuous version of the random field $\mathbf{F}$; according to Kunita [19] (Theorem 3.1.2 p.75), this property is true when the local characteristics $(\phi, \psi)$ are locally $\delta$-Hölder for some $\delta>0$ by Kolmogorov's criterion.

(ii) The second one is relative to differential properties: even if the random field $\mathbf{F}$ and its local characteristics $(\phi, \psi)$ are differentiable, it is not enough (as is shown in H. Kunita [19]), to get that the local characteristics of the derivative random field $\mathbf{F}_{x}$ are $\left(\phi_{x}, \psi_{x}\right)$.

(iii) Based on the study of Section 1 , we also need under which assumptions on the coefficients of a SDE, the solution is a regular monotonic random field with respect to its initial condition.

We start with a more precise definition of regular random field spaces than in Section 10 by introducing Hölder properties. The motivation is find in the Kolmogorov's continuity criterion (19]Theorem 1.4.1).

Theorem 2.1. Let $X(x), x \in \mathbb{D}$ a random field with values in a Banach space $B$, where $\mathbb{D}$ is a domain in $\mathbb{R}^{d}$. Assume that there exist positive constants $\gamma, C$ and $\alpha_{i}, i=1 \ldots d$ with $\sum_{i=1}^{d} \alpha_{i}^{-1}<1$, satisfying,

$$
\mathbb{E}\left[\|X(x)-X(y)\|^{\gamma}\right] \leq C\left(\sum_{i=1}^{d}\left\|x_{i}-y_{i}\right\|^{\alpha_{i}}\right), \quad \text { for any } x, y \in \mathbb{D}
$$

(i) Then $X(x)$ has a continuous modification $\widetilde{X}(x)$.

(ii) Let $0<\beta_{i} \leq A \alpha_{i}, i=1 \ldots d$ arbitrary positive numbers where $\left.A=\left(\alpha_{0}-d\right) / \gamma \alpha_{0}\right)$, and $\alpha_{0}^{-1} d=\sum_{i=1}^{d} \alpha_{i}^{-1}$. Then, for any hypercube $\mathbb{H}$ there exists a positive random variable $K(\omega)$ with $\mathbb{E}\left[K^{\gamma}\right]<\infty$ such that $\|X(x)-X(y)\|(\omega) \leq K(\omega)\left(\sum_{i=1}^{d}\left\|x_{i}-y_{i}\right\|^{\beta_{i}}\right) \quad$ for any $x, y \in \mathbb{H}$, a.s.. 
Recently, new criteria based on different norms, or chaining methods have been developed to weaken the criterion. A interesting survey may be find in Scheutzow [35].

\subsection{Regular Random Fields Spaces}

We introduce a family of Sobolev type random semi-norms to control locally or globally the growth of the random field and its derivatives.

Norms definition Let $\phi$ be a continuous $\mathbb{R}^{k}$-valued progressive random field and let $m$ be a non-negative integer, and $\delta$ a number in $(0,1]$. We need to control the asymptotic behavior in 0 and $\infty$ of $\phi$, and the regularity of its Hölder derivatives when there exist. More precisely, let $\phi$ be in the class $\mathcal{C}^{m, \delta}(] 0,+\infty[)$, i.e. $(m, \delta)$-times continuously differentiable in $x$ for any $t$, a.s.

(i) For any subset $K \subset] 0,+\infty$ [, we define the family of random (Hölder) $K$-semi-norms

$$
\left\{\begin{array}{l}
\|\phi\|_{m: K}(t, \omega)=\sup _{x \in K} \frac{\|\phi(t, x, \omega)\|}{x}+\sum_{1 \leq j \leq m} \sup _{x \in K}\left\|\partial_{x}^{j} \phi(t, x, \omega)\right\| \\
\|\phi\|_{m, \delta: K}(t, \omega)=\|\phi\|_{m: K}(t, \omega)+\sup _{x, y \in K} \frac{\left\|\partial_{x}^{m} \phi(t, x, \omega)-\partial_{x}^{m} \phi(t, y, \omega)\right\|}{|x-y|^{\delta}} .
\end{array}\right.
$$

The case $(m=0, \delta=1)$ corresponds to the local version of the Lipschitz case used in Section 1. When $K$ is all the domain $] 0,+\infty\left[\right.$, we simply write $\|\cdot\|_{m}(t, \omega)$, or $\|\cdot\|_{m, \delta}(t, \omega)$.

(ii) The first term of these random semi-norms differs slightly from the definition of Kunita seminorms (Equations (1) and (2) p.72) because instead of dividing by $1+|x|$ we divide by $x$ on the first terms. This does not change Kunita's results, but allows us to obtain reasonable behavior in the neighborhood of $x=0$ (Equation (1.7), Theorem [1.2) in addition to the traditional results in the neighborhood of $x=\infty$.

Different spaces of regular random fields The previous semi-norms are related to the spatial parameter. As in Definition 1.1, we add the temporal dimension in assuming these seminorms (or the square of the semi-norm) to be integrable in time with respect to the Lebesgue measure on $[0, T]$ for all $T$. Then, as in Lebesgue's Theorem, we can differentiate, pass to the limit, commute limit and integral for the random fields. Calligraphic notation recalls that these semi-norms are random.

(i) $\mathcal{K}_{l o c}^{m, \delta}\left(\right.$ resp. $\left.\overline{\mathcal{K}}_{l o c}^{m, \delta}\right)$ denotes the set of all $\mathcal{C}^{m, \delta}$-random fields such that for any compact $K \subset] 0,+\infty\left[\right.$, and any $T, \int_{0}^{T}\|\phi\|_{m, \delta: K}(t, \omega)<\infty,\left(\operatorname{resp} \cdot \int_{0}^{T}\|\psi\|_{m, \delta: K}^{2}(t, \omega) d t<\infty\right)$.

(ii) When these different norms are well-defined on the whole space ]0, $+\infty[$, the derivatives (up to a certain order) are bounded in the spatial parameter, with integrable (resp.square integrable) in time random bound. In this case, we use the notations $\mathcal{K}_{b}^{m}, \overline{\mathcal{K}}_{b}^{m}$ or $\mathcal{K}_{b}^{m, \delta}, \overline{\mathcal{K}}_{b}^{m, \delta}$.

\subsection{Differentiability of Itô random fields}

We shall discuss the regularity of a Itô semimartingale random field

$$
\mathbf{F}(\mathbf{t}, \mathbf{x})=\mathbf{F}(\mathbf{0}, \mathbf{x})+\int_{0}^{\mathbf{t}} \phi(\mathbf{s}, \mathbf{x}) \mathbf{d} \mathbf{s}+\int_{0}^{\mathbf{t}} \psi(\mathbf{s}, \mathbf{x}) \cdot \mathbf{d} \mathbf{W}_{\mathbf{s}}
$$

in connection with the regularity of its local characteristics $(\phi, \psi)$. As in Section 1, by convention, an Itô random field $\mathbf{F}$ is said to be a $\mathcal{K}_{l o c}^{m, \delta}$-semimartingale, whenever $F(0, x)$ is of class $\mathcal{C}^{m, \delta}$, 
$B^{F}(t, x)=\int_{0}^{t} \phi(s, x) d s$ is of class $\mathcal{K}_{l o c}^{m, \delta}$, and $M^{F}(t, x)=\int_{0}^{t} \psi(s, x) \cdot d W_{s}$ is of class $\overline{\mathcal{K}}_{l o c}^{m, \delta}$. The reference to $\mathcal{K}^{m, \delta}$ recall that $\mathbf{F}$ is a random field. 11 As in Kunita [19], we are concerned both by the regularity of $F$ from the regularity of its local characteristics $(\phi, \psi)$ (Theorem 3.1.2) and by the regularity of $(\phi, \psi)$ from that of $\mathbf{F}(\mathbf{t}, \mathbf{x})$ (Theorem 3.1.3). To be concise, we also give a sufficient conditions ([19] Theorem 3.3.3) under which we can differentiate term by term the dynamics of an Itô random field. This property is used in order to apply Itô-Ventzel's formula.

Theorem 2.2 (Differential Rules). Let $\mathbf{F}$ be an Itô semimartingale random field with local characteristics $(\phi, \psi), F(t, x)=F(0, x)+\int_{0}^{t} \phi(s, x) d s+\int_{0}^{t} \psi(s, x) . d W_{s}$

(i) If $\mathbf{F}$ is a $\mathcal{K}_{\text {loc }}^{m, \delta}$-semimartingale for some $m \geq 0, \delta \in(0,1]$, its local characteristics $(\phi, \psi)$ are of class $\mathcal{K}_{\text {loc }}^{m, \varepsilon} \times \overline{\mathcal{K}}_{\text {loc }}^{m, \varepsilon}$ for any $\varepsilon<\delta$.

(ii) Conversely, if the local characteristics $(\phi, \psi)$ are of class $\mathcal{K}_{l o c}^{m, \delta} \times \overline{\mathcal{K}}_{\text {loc }}^{m, \delta}$, then $\mathbf{F}$ is a $\mathcal{K}_{l o c}^{m, \varepsilon}$ semimartingale for any $\varepsilon<\delta$.

(iii) In any cases, for $m \geq 1, \delta \in(0,1]$, the derivative random field $\mathbf{F}_{x}$ is an Itô random field with local characteristics $\left(\phi_{x}, \psi_{x}\right)$.

(iv) Moreover, if $\mathbf{F}$ is a $\mathcal{K}_{\text {loc }}^{1, \delta} \cap \mathcal{C}^{2}$-semimartingale, for any Itô process $X, F(., X$.) is a continuous Itô semimartingale satisfying the Itô-Ventzel formula (1.10).

As previously mentioned, we also need results on the existence and the regularity of one dimensional random fields which are also solutions of stochastic differential equations (SDE). The spatial parameter in this case corresponds to the initial condition. Such random fields are also called stochastic flows and are the main subject (in the multidimensional case) of the Kunita's book [19].

The question is now to make assumptions on the coefficients in place of local characteristics. An example was given in Section 1, Theorem 1.2, where the global Lipschitz regularity of coefficients is used in proving the existence of monotonic solution. Next proposition, ([19] Theorem 4.6.5), frequently used in the sequel, extends these results to differentiability properties.

Proposition 2.3. Let $\mu$ be a real valued process and $\sigma$ be a d-dimensional process of the the class $\mathcal{K}_{\mathbf{b}}^{m, \delta}$ and $\overline{\mathcal{K}}_{\mathbf{b}}^{m, \delta}$ for some $m \geq 1, \delta \in(0,1]$. Consider the following $\operatorname{SDE}(\mu, \sigma)$,

$$
d X_{t}=\mu\left(t, X_{t}\right) d t+\sigma\left(t, X_{t}\right) \cdot d W_{t}, \quad X_{0}=x
$$

Then, in addition to the results of Theorem $1.2\left((\mu, \sigma) \in \mathcal{K}_{\mathbf{b}}^{0,1} \times \overline{\mathcal{K}}_{\mathbf{b}}^{0,1}\right)$ on the existence and uniqueness of global monotonic solution, we have:

(i) The unique solution $\mathbf{X}=\left(X_{t}^{x}, x>0\right)$ is a $\mathcal{K}_{\text {loc }}^{m, \varepsilon}$ semimartingale for any $\varepsilon<\delta$. The inverse $\mathbf{X}^{-1}$ of $\mathbf{X}$ is also of class $\mathcal{C}^{m}$.

(ii) $\mathbf{X}$ is strictly increasing in $x$, and its derivative $\mathbf{X}_{\mathbf{x}}$ is solution of a linear equation, with

\footnotetext{
${ }^{1}$ Note, in the case of general semimartingales $F$ studied by Kunita, the notion of local characteristics makes reference to the triplet $\left(\phi(t, x), a(t, x, y), A_{t}\right)$ for $x, y>0$, where the joint quadratic variation of $F(t, x), F(t, y)$ satisfy the relation $<F(t, x), F(t, y)>=\int_{0}^{t} a(s, x, y) d A_{s}$ with the process $A$ is $\mathcal{F}$-adapted increasing and continuous. Here, as there is a finite number of Brownian motions, $A_{t}=t$ and $a(t, x, y):=\psi(t, x) . \psi(t, y)$ where, as before, the "." denote the inner scalar product. From this, all assumptions on the matrix " $a$ " (in Kunita) are adapted to the present framework and replaced by our equivalent hypothesis on the diffusion characteristic vector $\psi$.
} 
spatially bounded stochastic parameters $\left(\mu_{x}\left(t, X_{t}^{x}\right), \sigma_{x}\left(t, X_{t}^{x}\right)\right)$ given by

$$
d X_{x}(t, x)=X_{x}(t, x)\left[\mu_{x}\left(t, X_{t}^{x}\right) d t+\sigma_{x}\left(t, X_{t}^{x}\right) \cdot d W_{t}\right], X_{x}(0, x)=1
$$

$\mathbf{1} / \mathbf{X}_{\mathbf{x}}$ is solution of the same kind of linear equation

$$
d Z_{t}=Z_{t}\left[\left(-\mu_{x}\left(t, X_{t}^{x}\right)+\left\|\sigma_{x}\left(t, X_{t}^{x}\right)\right\|^{2}\right) d t-\sigma_{x}\left(t, X_{t}^{x}\right) \cdot d W_{t}\right]
$$

Then, $\mathbf{X}_{\mathbf{x}}$ and $\mathbf{1} / \mathbf{X}_{\mathbf{x}}$ are $\mathcal{K}_{\text {loc }}^{m-1, \varepsilon}$-semimartingales.

(iii) The local characteristics of $\mathbf{X}, \lambda^{X}(t, x)=\mu\left(t, X_{t}^{x}\right)$ and $\theta^{X}(t, x)=\sigma\left(t, X_{t}^{x}\right)$ have only local properties and belong to $\mathcal{K}_{\text {loc }}^{m, \varepsilon} \times \overline{\mathcal{K}}_{\text {loc }}^{m, \varepsilon}$ for any $\varepsilon<\delta$.

Remark . This technical result shows clearly the interest of using Hölder property: the solution is fractionally less regular than the coefficients (going from $\delta$ in $\varepsilon<\delta$ ). Otherwise, if we are only interested with processes of class $\mathcal{K}^{m}$ ( $m$ integer) without worrying about the Hölder's dimension, then we will lose a hole order in the regularity: instead of solution of class $\mathcal{K}_{\text {loc }}^{m, \varepsilon}$, we will only obtain solution of class $\mathcal{K}_{l o c}^{m-1}$.

In the last proposition, we have made global regularity assumptions on the coefficients $(\mu, \sigma)$ in order to prove that the SDE (2.3) has a regular, non-exploding (global), strictly monotonic solution $X$. Observe that the solution itself as random field is only in $\mathcal{K}_{l o c}^{m, \varepsilon}$ in general. In Section 11. Paragraph 1.3, we have shown that even if the progressive marginal utility satisfies a $\operatorname{SDE}(\mu, \sigma)$ with global regularity, the $\operatorname{SDE}(\tilde{\mu}, \tilde{\sigma})$ satisfied by the inverse $-\widetilde{U}_{y}$ of $U_{x}$, assumed to be an Itô process, has only local regularity. So, we are also concerned with SDE whose the coefficients are of class $\mathcal{K}_{l o c}^{0,1} \times \overline{\mathcal{K}}_{l o c}^{0,1}$ or $\left.\left.\mathcal{K}_{l o c}^{m, \delta} \times \overline{\mathcal{K}}_{l o c}^{m, \delta}, m \geq 1, \delta \in\right] 0,1\right]$. Then, the SDE can not have global solution and explosion can occur at finite time $\zeta(x)$. Nevertheless, several properties are maintained as shown in 19] Theorems 4.7.1 and 4.7.2.

Theorem 2.4 (Local assumptions). Let us consider a SDE with only locally Lipschitz coefficients $\left((\mu, \sigma) \in \mathcal{K}_{\text {loc }}^{0,1} \times \overline{\mathcal{K}}_{\text {loc }}^{0,1}\right)$.

(i) For any initial condition $x$, the SDE has a unique maximal solution $\left(X_{t}^{x}\right)$ up to an explosion time $\zeta(x)$, that is on $[0, \zeta(x))$,

$$
d X_{t}^{x}=\mathbf{1}_{\{t<\zeta(x)\}} \mu\left(t, X_{t}^{x}\right) d t+\mathbf{1}_{\{t<\zeta(x)\}} \sigma\left(t, X_{t}^{x}\right) d W_{t}, \quad \text { a.s. }
$$

(ii) For given $t$, as function of $x, X_{t}^{x}$ is defined on its domain $\mathcal{D}_{t}=\{x: \zeta(x)>t\}$, with finite values on its range $\mathcal{R}_{t}(\omega)=\left\{X_{t}^{x}(\omega) ; x \in \mathcal{D}_{t}(\omega)\right\}$. Furthermore, $x \mapsto X_{t}^{x}: \mathcal{D}_{t} \rightarrow \mathcal{R}_{t}$ is a continuous strictly monotonic random field with continuous inverse $X_{t}^{-1}: \mathcal{R}_{t} \rightarrow \mathcal{D}_{t}$.

(iii) $\left(X_{t}^{x}\right)$ is a global solution (Theorem 1.2) if and only if the explosion time $\zeta(x)$ is equal to $\infty$ for all $x \in \mathbb{R}^{+}=[0, \infty)$ a.s., (or equivalently if for any time $t$, the domain $\mathcal{D}_{t}$ is the whole space $\mathbb{R}^{+}$a.s.). Hence the range $\mathcal{R}_{t}$ is also the whole space $\mathbb{R}^{+}$for any $t$.

(iv) Moreover, if $(\mu, \sigma) \in \mathcal{K}_{l o c}^{m, \delta} \times \overline{\mathcal{K}}_{\text {loc }}^{m, \delta}(m \geq 1, \delta \in(0,1]) X_{t}($.$) is of class \mathcal{C}^{m, \varepsilon}, \varepsilon<\delta$ on $\mathcal{D}_{t}$.

(v) If $\left(X_{t}^{x}\right)$ is a global solution, then all assertions of Proposition 2.3 hold true.

We recall the proof of Kunita to present the truncation method which is the base of many proofs when only local properties hold true. 
Proof. (i) Following Kunita, we shall apply the method of truncation on $\mathbb{R}^{+}$. For each positive integer $N$, take a $\mathcal{C}^{\infty}$-function $\psi^{N}(x), x \in \mathbb{R}^{+}$such that $\psi^{N}(x)=1$ if $x \leq N, 0 \leq \psi^{N}(x) \leq 1$ if $N \leq x \leq N+1$ and $\psi^{N}(x)=0$ if $x>N$. Define $\mu^{N}(t, x)=\mu(t, x) \psi^{N}(x)$ and $\sigma^{N}(t, x)=$ $\sigma(t, x) \psi^{N}(x)$ which belong to $\mathcal{L}_{b}^{m, \delta}$ and $\overline{\mathcal{K}}_{b}^{m, \delta}$. Therefore, the $\operatorname{SDE}\left(\mu^{N}, \sigma^{N}\right)$ has a unique solution $X^{N}$ of class $\mathcal{K}^{m, \epsilon}(\epsilon<\delta)$ satisfying assertions of Proposition 2.3. Let us introduce the family of stopping times for each $x, \zeta_{N}(x):=\inf \left\{t: X^{N}(t, x) \geq N\right\}$. By uniqueness, for $M<N$ the solutions $X^{M}(x)$ and $X^{N}(x)$ coincide on $\left[0, \zeta_{M}(x)\right)$. Then, the family $\zeta_{N}(x)$ is increasing with limit $\zeta(x):=\lim _{N \rightarrow \infty} \zeta_{N}(x)$. We can define $X_{t}(x), t<\zeta(x)$ by $X_{t}(x)=X_{t}^{N}(x)$ if $t<\zeta_{N}(x)$. It is a maximal solution of $\operatorname{SDE}(\mu, \sigma)$ starting at $x$ at $t=0$.

(ii) We shall prove that the above $X .(x)$ is a continuous strictly increasing flow. Take any sample $\omega$ such that $X_{t}^{N}(\omega,$.$) defines a continuous map strictly increasing in x$ for any $N$. Probability of the set of all such samples is 1 . We first note that each $\zeta_{N}(\omega, x)$ is lower semicontinuous in $x$ i.e. $\mathcal{D}_{t}^{N}(\omega)=\left\{x: \zeta_{N}(\omega, x)>t\right\}$ is open for any $t>0$, since on this set $X_{s}^{N}(\omega, x)<N$ for all $s \leq t$. Hence, the same inequality holds for any $x^{\prime}$ in the neighborhood of $x$. Now since $\zeta(\omega, x)$ is the upper limit of $\zeta_{N}(\omega, x)$ it is also lower semicontinuous. Set $\mathcal{D}_{t}(\omega)=\cup_{N} \mathcal{D}_{t}^{N}(\omega)$. Since $X(t, x)=X^{N}(t, x)$ holds on $\mathcal{D}_{t}^{N}(\omega)$, the map $X(t, x): \mathcal{D}_{t}(\omega) \rightarrow \mathbb{R}^{+}$is continuous strictly increasing with continuous inverse.

(iii) The differentiability properties are showed similarly by truncation techniques, using derivability of processes $X^{N}$.

Comment Recently, several papers address the question: under which minimal assumptions on the SDE's coefficients, the solution is non-explosive? For example, when the coefficients $\mu$ and $\sigma$ are a deterministic functions independent of the time, the property holds true under global log-Lipschitz type conditions as it is showed in Zongxia [20] and Fang [12]. But these new results and many others can not be applied directly to our study because the coefficients of SDE's we are concerned are structurally stochastic.

Local regularity on SDEs coefficients appears as a kind of minimal assumption to ensure the regularity of a global solution if there exists. Because of its importance in the sequel, we give a name to this class of SDE's.

Definition 2.1. A $\operatorname{SDE}(\mu, \sigma)$ is said to be of class $\mathcal{S}^{m, \delta}$ if

a) the coefficients $(\mu, \sigma)$ are in the spaces $\left.\left(\mathcal{K}_{l o c}^{m, \delta}, \overline{\mathcal{K}}_{\text {loc }}^{m, \delta}\right)\right)$

b) the maximal solution $X$ is non explosive.

By Theorem 2.4, the unique solution $X$ is strictly monotonic with range $[0, \infty)$ and of class $\mathcal{K}_{l o c}^{m, \varepsilon}, \varepsilon<\delta$.

Classical examples of $\mathcal{S}^{m, \delta}$ SDEs are given by $\operatorname{SDE}(\mu, \sigma)$ when $(\mu, \sigma)$ are in the spaces $\left(\mathcal{K}_{b}^{m}, \overline{\mathcal{K}}_{b}^{m}\right)$, or even in $\left(\mathcal{K}_{b}^{0}, \overline{\mathcal{K}}_{b}^{0}\right) \cap\left(\mathcal{K}_{\text {loc }}^{m, \delta}, \overline{\mathcal{K}}_{\text {loc }}^{m, \delta}\right)$. Moreover, in these last two cases, the asymptotic behavior of the solution is given by Theorem 1.2 Equation (1.6) and Equation (1.7).

\subsection{Solvable SPDEs via SDEs}

Dynamics of inverse flow of regular SDE solution In the utility framework, under the strong assumptions of Theorem 1.5, we have shown that $\widetilde{U}_{y}$, the inverse flow of $\left(-U_{x}(t, x)\right)$, is solution of a $\operatorname{SPDE}$ and a $\operatorname{SDE}(\tilde{\mu}, \tilde{\sigma})$ simultaneously. We want to relax the a priori assumption 
that the inverse flow $\widetilde{U}_{y}$ is an Itô random field. So, we proceed differently, by starting from the maximal solution of the local SDE $(\tilde{\mu}, \tilde{\sigma})$ with explosion time $\zeta(x)$, and by verifying that up to $\zeta(x)$ this process is the inverse flow of the solution of the $\operatorname{SDE}(\mu, \sigma)$. By an easy argument base on the uniqueness of non-explosive solution of the $\operatorname{SDE}(\mu, \sigma)$, we deduce $\zeta(x)=\infty$, a.s.. Some regularity on the $\operatorname{SDE}(\mu, \sigma)$ is required to conduct calculation and conclude.

Theorem 2.5 (Inverse flow SDE). Let $\left(X_{t}^{x}\right)$ be the monotonic solution of a $S D E(\mu, \sigma)$ of class $\left.\left.\mathcal{S}^{m, \delta}, m \geq 3, \delta \in\right] 0,1\right]$, so that as random field $\left(X_{t}^{x}\right)$ and its local characteristics $\left(\lambda(t, x)=\mu\left(t, X_{t}^{x}\right)\right)$ and $\left(\theta(t, x)=\sigma\left(t, X_{t}^{x}\right)\right)$ are of class $\mathcal{K}_{\text {loc }}^{m, \varepsilon}$ and $\mathcal{L}_{\text {loc }}^{m, \varepsilon} \times \overline{\mathcal{K}}_{\text {loc }}^{m, \varepsilon}$ for any $0<\varepsilon<1$.

We are concerned with the $S D E(\tilde{\mu}, \tilde{\sigma})$

$$
d \xi_{t}=-\frac{1}{X_{x}\left(t, \xi_{t}\right)}\left[\left(\lambda\left(t, \xi_{t}\right)-\frac{1}{2} \partial_{x}\left(\frac{\|\theta\|^{2}}{X_{x}}\right)\left(t, \xi_{t}\right)\right) d t+\theta\left(t, \xi_{t}\right) . d W_{t}\right], \quad \xi_{0}=z,
$$

where $\tilde{\sigma}(t, z)=-\frac{\theta(t, z)}{X_{x}(t, z)}$ and $\tilde{\mu}(t, z)=\frac{1}{X_{x}(t, z)}\left(\frac{1}{2} \partial_{x}\left(\frac{\|\theta\|^{2}}{X_{x}}\right)(t, z)-\lambda(t, z)\right)$.

(i) The $\operatorname{SDE}(\tilde{\mu}, \tilde{\sigma})$ is of class $\mathcal{S}^{m-2, \varepsilon}(0<\varepsilon<\delta)$ and its unique monotonic solution $\xi^{z}$ is the inverse flow $X^{-1}$ of $X$.

(ii) Consequently, the inverse $X^{-1}$ of $X$ is a semimartingale and belongs to the class $\mathcal{K}_{\text {loc }}^{m-2, \varepsilon} \cap \mathcal{C}^{m}$.

Proof. The proof is in several steps, by first proving the local regularity of the coefficients $(\tilde{\mu}, \tilde{\sigma})$, and then the existence of a monotonic solution $\xi^{z}$ up to a explosion time $\zeta(z)$. The main step is then to prove that $\zeta(z)=\infty$ a.s. by showing that locally $\xi^{z}$ is the inverse $X^{-1}$.

(i) Since $X \in \mathcal{K}_{l o c}^{m, \varepsilon}$ and $(\lambda, \theta) \in \mathcal{K}_{l o c}^{m, \varepsilon} \times \overline{\mathcal{K}}_{l o c}^{m, \varepsilon}$, by Proposition $2.31 / X_{x} \in \mathcal{K}_{l o c}^{m-1, \varepsilon}$ and so $\left(\lambda / X_{x}, \theta / X_{x}\right) \in \mathcal{K}_{l o c}^{m-1, \varepsilon} \times \overline{\mathcal{K}}_{l o c}^{m-1, \varepsilon}$ and $\partial_{x}\left(\frac{\|\theta\|^{2}}{X_{x}}\right) \in \mathcal{K}_{l o c}^{m-2, \varepsilon}$. Consequently the coefficients $(\tilde{\mu}, \tilde{\sigma})$ are of class $\mathcal{K}_{l o c}^{m-2, \varepsilon} \times \overline{\mathcal{K}}_{l o c}^{m-1, \varepsilon}$.

(ii) Since the coefficients $(\tilde{\mu}, \tilde{\sigma})$ satisfy the assumptions of Theorem 2.4 the $\operatorname{SDE}(\tilde{\mu}, \tilde{\sigma})$ has a unique maximal solution $\xi^{z}$, up to an explosion time $\zeta(z)$.

a) We claim that the solution $\xi_{t}^{z}$ is the inverse flow $X^{-1}(t, z)$ for $t \in[0, \zeta(z))$ of the $\operatorname{SDE}(\mu, \sigma)$ monotonic solution $X_{t}^{x}:=X(t, x)$. Since by assumption $X$ is of class $\mathcal{K}_{l o c}^{m, \epsilon}$ and its local characteristics $(\lambda, \theta)$ are of class $(\lambda, \theta) \in \mathcal{K}_{l o c}^{m, \varepsilon} \times \overline{\mathcal{K}}_{l o c}^{m, \varepsilon}(m \geq 3, \epsilon \in] 0, \delta[)$, we can apply Itô-Ventzel's formula to $X\left(t, \xi_{t}^{z}\right)$ up to the time $\zeta(z)$. Then, on $[0, \zeta(z))$, by using the short notation $\xi$ in place of $\xi^{z}$,

$$
\begin{aligned}
d X\left(t, \xi_{t}\right) & =\left[\theta\left(t, \xi_{t}\right)+X_{x}\left(t, \xi_{t}\right) \frac{-\theta\left(t, \xi_{t}\right)}{X_{x}\left(t, \xi_{t}\right)}\right] \cdot d W_{t} \\
& +\left[\lambda\left(t, \xi_{t}\right)+X_{x}\left(t, \xi_{t}\right)\left(-\frac{1}{X_{x}\left(t, \xi_{t}\right)}\left(\lambda\left(t, \xi_{t}\right)-\frac{1}{2} \partial_{x}\left(\frac{\|\theta\|^{2}}{X_{x}}\right)\left(t, \xi_{t}\right)\right)\right)\right] d t \\
& +\left[\frac{1}{2} X_{x x}\left(t, \xi_{t}\right)\left(\frac{\|\theta\|^{2}}{X_{x}^{2}}\right)\left(t, \xi_{t}\right)+\left(\theta_{x}\left(t, \xi_{t}\right) \cdot\left(\frac{-\theta}{X_{x}}\right)\left(t, \xi_{t}\right)\right] d t\right. \\
& \left.=[0] \cdot d W_{t}+\left[\frac{1}{2} \partial_{x}\left(\frac{\|\theta\|^{2}}{X_{x}}\right)\left(t, \xi_{t}\right)-\frac{1}{2}\left(\frac{1}{X_{x}} \partial_{x}\|\theta\|^{2}\right)\left(t, \xi_{t}\right)\right)-\frac{1}{2}\left(\partial_{x}\left(\frac{1}{X_{x}}\right)\|\theta\|^{2}\right)\left(t, \xi_{t}\right)\right] d t \\
& =0
\end{aligned}
$$

b) Then the continuous (in time) process $X\left(t, \xi_{t}^{z}\right)$ is constant a.s. on $[0, \zeta(z))$. At time $t=\zeta(z)<$ $\infty, \xi_{t}^{z}=\infty$ and $X(t, \infty)=\infty$. On the other hand, by continuity, $X\left(t, \xi_{t}^{z}\right)=z$ if $t=\zeta(z)<\infty$. To avoid contradiction, necessarily $\zeta(z)=\infty$, a.s.. 
As in Theorem 1.5, we can also characterize the inverse process in terms of monotonic solution of non-linear stochastic partial differential equation (SPDE). This point of view is well-suited to the study of consistent dynamic utilities developed in the next sections.

Solvable SPDEs via SDEs Now we show how to solve some non-linear SPDEs via SDEs. A first link is obtained by the transformation of the SDE (2.7) into a SPDE. Recall that conditions of type $\mathcal{S}^{m, \delta}$ are related to the coefficients of the SDE, when conditions of type $\mathcal{K}_{\text {loc }}^{m, \delta}$ are related to the local characteristics of the solution.

Theorem 2.6 (SPDE point of view). Let us consider a $\operatorname{SDE}(\mu, \sigma)$ of class $\mathcal{S}^{m, \delta}$ with $m \geq$ $2, \delta \in(0,1]$, and its adjoint operator $\widehat{L}_{t, z}^{\sigma, \mu}=\frac{1}{2} \partial_{z}\left(\|\sigma(t, z)\|^{2} \partial_{z}\right)-\mu(t, z) \partial_{z}$. Denote by $X$ its unique solution.

(i) For $m \geq 3$, the inverse flow $X^{-1}=\xi^{X}$ of $X$ is a strictly monotonic solution of class $\mathcal{K}_{\text {loc }}^{m-2, \delta} \cap \mathcal{C}^{m}$ of $\operatorname{SPDE}\left(\widehat{L}^{\sigma, \mu},-\sigma \partial_{z}\right)$, with initial condition $\xi_{0}(z)=z$,

$$
d \xi(t, z)=-\xi_{z}(t, z) \sigma(t, z) \cdot d W_{t}+\widehat{L}_{t, z}^{\sigma, \mu}(\xi) d t
$$

(ii) Conversely, $(m \geq 2)$, let $\xi$ be a $\mathcal{K}_{\text {loc }}^{1, \delta} \cap \mathcal{C}^{2}$-regular solution of $\operatorname{SPDE}\left(\widehat{L}^{\sigma, \mu},-\sigma \partial_{z}\right)$ (2.8). Then, $\xi(t, X(t, x)) \equiv x$ and $\xi$ is the strictly monotonic inverse flow $X^{-1}:=\xi^{X}$ of $X$. Moreover, uniqueness holds true for the $\operatorname{SPDE}\left(\widehat{L}^{\sigma, \mu},-\sigma \partial_{z}\right)$ in the class of $\mathcal{K}_{\text {loc }}^{1, \delta} \cap \mathcal{C}^{2}$-regular solutions.

Comment When the coefficients $(\mu, \sigma)$ of the SDE are non random, in a multi-dimensional case, the $\operatorname{SPDE}\left(\widehat{L}^{\sigma, \mu},-\sigma \partial_{z}\right)$ is known as the stochastic transport equation in Zhang [40] or in Constantin and Iyer $[3]$.

Proof. (i) According to notations of Theorem 2.5 since $\xi^{X}$ is the inverse of $X$, we have:

$$
\begin{aligned}
& \lambda\left(t, \xi^{X}(t, z)\right)=\mu\left(t, X\left(t, \xi^{X}(t, z)\right)\right)=\mu(t, z), \quad \xi_{z}^{X}(t, z)=\frac{1}{X_{x}\left(t, \xi^{X}(t, z)\right)}, \\
& \theta\left(t, \xi^{X}(t, x)\right)=-\frac{\sigma\left(t, X\left(t, \xi^{X}(t, z)\right)\right)}{X_{x}\left(t, \xi^{X}(t, z)\right)}=-\xi_{z}^{X}(t, z) \sigma(t, z)
\end{aligned}
$$

which easily leads to $\tilde{\mu}\left(t, \xi^{X}(t, z)\right)=\widehat{L}_{t, z}^{\sigma, \mu}\left(\xi^{X}\right)(t, z)$. So $\xi^{X}$ is solution of the SPDE (2.8).

(ii) Let now turn to the converse implication by starting from a given monotonic solution $\xi$ of class $\mathcal{K}_{l o c}^{1, \delta} \cap \mathcal{C}^{2}$ of the $\operatorname{SPDE}: d \xi(t, z)=-\xi_{z}(t, z) \sigma(t, z) \cdot d W_{t}+\widehat{L}_{t, z}^{\sigma, \mu}(\xi) d t$.

a) From Theorem 2.2, $\xi$ is regular enough to use Itô-Ventzel's formula with the solution $X(t, x)=$ $X_{t}^{x}$ of the $\operatorname{SDE}(\mu, \sigma)$ to compute the dynamics of $H(t, x)=\xi(t, X(t, x))$. In the next equation, we do not recall the parameter $x$.

$$
\begin{aligned}
d H_{t} & =\left(-\xi_{z}\left(t, X_{t}\right) \sigma\left(t, X_{t}\right)-\xi_{z}\left(t, X_{t}\right) \sigma\left(t, X_{t}\right)\right) \cdot d W_{t} \\
& +\left(\widehat{L}^{\sigma, \mu}(\xi)+\frac{1}{2} \xi_{z z}\|\sigma\|^{2}+\mu \xi_{z}+\partial_{z}\left(-\xi_{z} \sigma\right) \cdot \sigma\right)\left(t, X_{t}\right) d t \\
& =\left(\xi_{z z}\|\sigma\|^{2}+\frac{1}{2} \xi_{z}\left(\partial_{z}\|\sigma\|^{2}\right)-\partial_{z}\left(\xi_{z}\right)\|\sigma\|^{2}-\frac{1}{2} \xi_{z}\left(\partial_{z}\|\sigma\|^{2}\right)\right)\left(t, X_{t}\right) d t \\
& =0
\end{aligned}
$$

The random field $H(t, x)=\xi(t, X(t, x))$ is constant in time and equal to its initial condition $x$. This finishes the proof that $X$ is the inverse flow of $\xi$. 
b) Since $\operatorname{SDE}(\mu, \sigma)$ is of $\mathcal{S}^{m, \delta}$, there is only one solution $X$. Then any "regular" solution $\xi$ of the SPDE is the inverse of $X$ and then is unique.

Next result, useful for applications, is a slight extension of the previous one. It establishes a connection between a more general second order SPDE and two SDEs. As discussed in the following, this connection is in the core of the study of consistent dynamic utilities. It is based on the observation that if $\xi$ is the inverse of $\operatorname{SDE}\left(\mu^{X}, \sigma^{X}\right)$ monotonic solution $X$ and if $\phi \in \mathcal{C}^{2}$ a regular monotonic function, the process $X(., \phi(x))$ satisfies the same $\operatorname{SDE}\left(\mu^{X}, \sigma^{X}\right)$, and so its inverse $\phi^{-1}(\xi .(z))$ satisfies the same SPDE than $\xi$. The extension, given in the following result describes the dynamics of compound processes $Y(t, \xi(t, z))$ and $\xi(t, \bar{X}(t, \bar{x})$ for regular Itô semimartingales $Y$ and $\bar{X}$ and show how to solve the associated SPDEs.

Theorem 2.7. Let $X$ be a solution of $S D E\left(\mu^{X}, \sigma^{X}\right)$ and $\xi$ a $\mathcal{K}_{\text {loc }}^{1, \delta} \cap \mathcal{C}^{2}$-regular solution $(\delta>0)$ of the $\operatorname{SPDE}\left(\widehat{L}^{X},-\sigma^{X} \partial_{z}\right)$, where $\widehat{L}_{t, z}^{X}=\widehat{L}_{t, z}^{\sigma^{X}, \mu^{X}}=\frac{1}{2} \partial_{z}\left(\left\|\sigma^{X}(t, z)\right\|^{2} \partial_{z}\right)-\mu^{X}(t, z) \partial_{z}$.

(i) Let $Y$ be a solution of class $\mathcal{K}_{\text {loc }}^{1, \delta} \cap \mathcal{C}^{2}$ of $S D E\left(\mu^{Y}, \sigma^{Y}\right)$ with initial condition $\phi \in \mathcal{C}^{2}$. Then the random field $Y(t, \xi(t, z))=G(t, z)$ evolves as,

$$
\begin{aligned}
d G(t, z) & =\sigma^{Y}(t, G(t, z)) \cdot d W_{t}+\mu^{Y}(t, G(t, z)) d t \\
& \left.-\partial_{z} G(t, z) \sigma^{X}(t, z)\left[d W_{t}+\sigma_{y}^{Y}(t, G(t, z)) d t\right]+\widehat{L}_{t, z}^{X}(G)(t, z)\right) d t
\end{aligned}
$$

(ii) Let $\bar{X}$ be a solution of $\operatorname{SDE}(\bar{\mu}, \bar{\sigma})$ with initial condition $\psi(\bar{x})$. Denote by $\Delta \mu(t, z):=\mu^{X}(t, z)-$ $\bar{\mu}\left(t, \bar{X}_{t}\right)$ and $\Delta \sigma(t, z):=\sigma^{X}(t, z)-\bar{\sigma}\left(t, \bar{X}_{t}\right)$. Then the random field $\xi\left(t, \bar{X}_{t}\right)$ evolves as

$$
d \xi\left(t, \bar{X}_{t}\right)=-\xi_{z}\left(t, \bar{X}_{t}\right)\left[\left(\sigma^{X}\left(t, \bar{X}_{t}\right)-\bar{\sigma}\left(t, \bar{X}_{t}\right)\right) \cdot\left(d W_{t}-\bar{\sigma}_{\bar{x}}\left(t, \bar{X}_{t}\right) d t\right)\right]+\widehat{L}^{\Delta}(\xi)\left(t, \bar{X}_{t}\right) .
$$

(iii) Solvable SPDE: Let $G$ be a solution of class $\mathcal{K}_{\text {loc }}^{1, \delta} \cap \mathcal{C}^{2}$-regular of the SPDE (2.9); then the process $G\left(t, \bar{X}_{t}(\bar{x})\right)$ with initial condition $G(0, \psi(\bar{x}))$ evolves as

$$
\begin{aligned}
d G\left(t, \bar{X}_{t}\right) & =\sigma^{Y}\left(t, G\left(t, \bar{X}_{t}\right)\right) \cdot d W_{t}+\mu^{Y}\left(t, G\left(t, \bar{X}_{t}\right)\right) d t \\
& -G_{z}\left(t, \bar{X}_{t}\right)\left(\sigma^{X}-\bar{\sigma}\right)\left(t, \bar{X}_{t}\right) \cdot\left[d W_{t}+\left(\sigma_{y}^{Y}(G)-\bar{\sigma}_{\bar{x}}\right)\left(t, \bar{X}_{t}\right) d t\right]+\widehat{L}^{\Delta}(G)\left(t, \bar{X}_{t}\right) d t
\end{aligned}
$$

(iv) In particular, $G\left(t, X_{t}(\phi(y))\right.$ is a solution of the $S D E\left(\mu^{Y}, \sigma^{Y}\right)$ with initial condition $\phi(y)$. If uniqueness holds true for this equation, then $G(t, z)=Y_{t}(t, \xi(t, z))$ and uniqueness also holds true for the SPDE (2.9).

Note the different nature of assumptions (which may be equivalent) in the assertions of this theorem. In (i), we assume that the coefficients are regular enough such that $Y$ satisfies the Itô-Ventzel assumptions and such that the inverse $\xi$ of $X$ is an Itô semimartingale, while in (ii) we only suppose the existence of $X$ (without regularity), but in return we assume the existence of a smooth solution $G$ of the SPDE (2.9).

Otherwise, remark that the first line in (2.9), associated with $Y$, is purely the SDE part of the dynamics of $G$ while the second corresponds to the partial differential part. This writing suggests a simple method for solving such equations. Indeed, when considering any SPDE, if we are able to rewrite it in the form (2.9), then we can hope solve it (if regularity of the identified coefficients holds) by associating two SDEs, and then by composing with the solution associated with the partial differential part ( $X$ in our result); this is the aim of assertions ((iii), (iv)). 
Proof. (i) The proof is based on the Itô-Ventzel formula, applied to $Y$ as random field, and $\xi(t, z)$ as semimartingale; that leads to

$$
\begin{aligned}
d G(t, z) & =\mu^{Y}(t, G(t, z)) d t+\left(\sigma^{Y}(t, G(t, z))-Y_{y}(t, \xi(t, z)) \xi_{z}(t, z) \sigma^{X}(t, z)\right) \cdot d W_{t} \\
& +\frac{1}{2} Y_{y y}(t, \xi(t, z))\left\|-\xi_{z}(t, z) \sigma^{X}(t, z)\right\|^{2}+X_{x}(t, \xi(t, y)) \widehat{L}_{t, z}^{Z}(\xi) d t \\
& +<d Y_{y}(t, y), d \xi(t, z)>\left.\right|_{y=\xi(t, z)} \\
& =\mu^{Y}(t, \xi(t, z)) d t+\left(\sigma^{Y}(., G)-Y_{y}(., \xi) \xi_{z} \sigma^{X}\right)(t, z) \cdot d W_{t} \\
& +\left[\frac{1}{2} Y_{y y}(., \xi)\left\|\xi_{z} \sigma^{X}\right\|^{2}+Y_{y}(., G) \widehat{L}^{X}(\xi)-G_{z} \sigma_{y}^{Y}(., G) \cdot \sigma^{X}\right](t, z) d t
\end{aligned}
$$

Now using identity $\partial_{z}\left(Y_{y}(t, \xi(t, z))\right)=Y_{y y}(t, \xi(t, z)) \xi_{z}(t, z)$ and $G_{z}(t, z)=Y_{y}(t, \xi(t, z)) \xi_{z}(t, z)$, it follows, at first, that

$$
\begin{aligned}
Y_{y y}(., \xi)\left\|-\xi_{z} \sigma^{X}\right\|^{2}+Y_{y}(., \xi) \partial_{z}\left(\left\|\sigma^{X}\right\|^{2} \xi_{z}\right) & \left.=\partial_{z}\left(Y_{y}(., \xi)\right)\left(\xi_{z}\left\|\sigma^{X}\right\|^{2}\right)+Y_{y}(., \xi) \partial_{z}\left(\left\|\sigma^{X}\right\|^{2} \xi_{z}\right)\right) \\
& =\partial_{z}\left(Y_{y}(., \xi) \xi_{z}\left\|\sigma^{X}\right\|^{2}\right)=\partial_{z}\left(\left\|\sigma^{X}\right\|^{2} G_{z}\right)
\end{aligned}
$$

Second, by injecting this identity in the dynamics of $G$, we obtain

$$
\begin{aligned}
d G(t, z) & =\left(\sigma_{t}^{Y}(G(t, z))-G_{z}(t, z) \sigma_{t}^{X}(z)\right) \cdot d W_{t} \\
& +\left(\widehat{L}_{t, z}^{X}(G)+\mu_{t}^{Y}(G(t, z))-\partial_{z}\left(\sigma^{Y}(t, G(t, z)) \cdot \sigma^{X}(t, z)\right)\right) d t
\end{aligned}
$$

In a simpler formulation, that is equivalent to,

$$
\begin{aligned}
d G(t, z) & =\sigma^{Y}(t, G(t, z)) \cdot d W_{t}+\mu^{Y}(t, G(t, z)) d t \\
& -\partial_{z} G(t, z) \sigma^{X}(t, z)\left[d W_{t}+\sigma_{y}^{Y}(t, G(t, z)]+\widehat{L}^{X}(G)(t, z) d t\right.
\end{aligned}
$$

In the particular case, where $Y(t, x)=F(x), \mu^{Y} \equiv 0$ and $\sigma^{Y} \equiv 0$ and the result is obvious.

(ii) Again, Itô-Ventzel calculus yields to

$$
\begin{aligned}
d \xi\left(t, \bar{X}_{t}\right) & =\left[\frac{1}{2} \partial_{y}\left(\left\|\sigma^{X}(t, y)\right\|^{2} \xi(t, y)\right)\left(t, \bar{X}_{t}\right)-\mu^{X}\left(t, \bar{X}_{t}\right) \xi_{z}\left(t, \bar{X}_{t}\right)\right] d t \\
& +\xi_{z}\left(t, \bar{X}_{t}\right) \bar{\sigma}\left(t, \bar{X}_{t}\right) d W_{t}+\left[\xi_{z}\left(t, \bar{X}_{t}\right) \mu^{X}\left(t, \bar{X}_{t}\right)+\frac{1}{2} \xi_{z z}\left(t, \bar{X}_{t}\right)\left\|\bar{\sigma}\left(t, \bar{X}_{t}\right)\right\|^{2}\right. \\
& \left.-\left(\xi_{y y}\left(t, \bar{X}_{t}\right) \sigma^{X}\left(t, \bar{X}_{t}\right)+\xi_{y}\left(t, \bar{X}_{t}\right) \sigma_{z}^{X}\left(t, \bar{X}_{t}\right)\right) \cdot \bar{\sigma}\left(t, \bar{X}_{t}\right)\right] d t \\
& -\xi_{z}\left(t, \bar{X}_{t}\right) \sigma^{X}\left(t, \bar{X}_{t}\right) d W_{t}
\end{aligned}
$$

and by arranging the terms properly we get

$$
\begin{aligned}
d \xi\left(t, \bar{X}_{t}\right) & =-\xi_{z}\left(t, \bar{X}_{t}\right)\left(\sigma^{X}\left(t, \bar{X}_{t}\right)-\bar{\sigma}\left(t, \bar{X}_{t}\right)\right) d W_{t}-\xi_{z}\left(t, \bar{X}_{t}\right)\left(\mu^{X}\left(t, \bar{X}_{t}\right)-\bar{\mu}\left(t, \bar{X}_{t}\right)\right) d t \\
& +\frac{1}{2} \xi_{z z}\left(t, \bar{X}_{t}\right)\left(\left\|\bar{\sigma}\left(t, \bar{X}_{t}\right)\right\|^{2}+\left\|\sigma^{X}\left(t, \bar{X}_{t}\right)\right\|^{2}-2 \sigma^{X}\left(t, \bar{X}_{t}\right) \cdot \bar{\sigma}\left(t, \bar{X}_{t}\right)\right) d t \\
& +\xi_{z}\left(t, \bar{X}_{t}\right)\left(\sigma^{X}\left(t, \bar{X}_{t}\right)-\bar{\sigma}\left(t, \bar{X}_{t}\right)\right) \cdot \sigma_{z}^{X}\left(t, \bar{X}_{t}\right) d t \\
& =-\xi_{z}\left(t, \bar{X}_{t}\right)\left(\sigma^{X}\left(t, \bar{X}_{t}\right)-\bar{\sigma}\left(t, \bar{X}_{t}\right)\right) d W_{t}-\xi_{z}\left(t, \bar{X}_{t}\right)\left(\mu^{X}\left(t, \bar{X}_{t}\right)-\bar{\mu}\left(t, \bar{X}_{t}\right)\right) d t \\
& +\frac{1}{2} \xi_{z z}\left(t, \bar{X}_{t}\right)\left\|\bar{\sigma}\left(t, \bar{X}_{t}\right)-\sigma^{X}\left(t, \bar{X}_{t}\right)\right\|^{2} d t \\
& +\xi_{z}\left(t, \bar{X}_{t}\right)\left(\sigma^{X}\left(t, \bar{X}_{t}\right)-\bar{\sigma}\left(t, \bar{X}_{t}\right)\right) \cdot \sigma_{x}^{X}\left(t, \bar{X}_{t}\right) d t
\end{aligned}
$$


Subsequently, by introducing new coefficients $\Delta \mu$ and $\Delta \sigma$ as follows:

$$
\Delta \mu(t, y):=\mu^{X}(t, y)-\bar{\mu}\left(t, \bar{X}_{t}\right) \text { et } \Delta \sigma(t, y):=\sigma^{X}(t, y)-\bar{\sigma}\left(t, \bar{X}_{t}\right)
$$

the last line becomes $\partial_{y}\left(\|\Delta \sigma(t, y)\|^{2} \xi_{z}\right)\left(t, \bar{X}_{t}\right)$ which yields to the desired formula:

$$
\begin{aligned}
d \xi\left(t, \bar{X}_{t}\right) & =-\xi_{z}\left(t, \bar{X}_{t}\right)\left(\sigma^{X}\left(t, \bar{X}_{t}\right)-\bar{\sigma}\left(t, \bar{X}_{t}\right)\right) d W_{t}+\widehat{L}^{\Delta}(\xi)\left(t, \bar{X}_{t}\right) d t \\
& =-\xi_{z}\left(t, \bar{X}_{t}\right) \hat{\sigma}\left(t, \bar{X}_{t}\right) d W_{t}+\widehat{L}^{\Delta}(\xi)\left(t, \bar{X}_{t}\right) d t
\end{aligned}
$$

(iii) The properties of the random field $G\left(t, \bar{X}_{t}\right)$ are obtained once again from Itô-Ventzel's formula, in a very similar way than for $\xi\left(t, \bar{X}_{t}\right)$, since the second line of Equation (2.9) has the same form than the SPDE of $\xi$ except that the Brownian $d W$ is replaced by $d W .+\sigma_{x}^{Y}(G) d t$. So we obtain Equation (2.11).

Back to Progressive Utilities Let now come back to progressive utilities $\mathbf{U}$. The results of this section will be of great use in the rest of this work, especially when we focus on dynamic optimal portfolios. We give sufficient conditions on progressive dynamic utilities so that assumptions of Theorem 1.1 are satisfied, in particular that the inverse $-\widetilde{U}_{y}$ of $U_{x}$ is a semimartingale. These assumptions are made on the coefficients of the intrinsic $\operatorname{SDE}(\mu, \sigma)$ and not on the local characteristics $(\beta, \gamma)$, since essential results are obtained from SDEs properties.

Theorem 2.8. Consider $\operatorname{SDE}(\mu, \sigma)$ of class $\mathcal{S}^{1, \delta}, \delta \in(0,1]$ and let $Z$ be its unique monotonic solution of class $\mathcal{K}_{\text {loc }}^{1, \varepsilon}$ for any $0<\varepsilon<\delta$. For any deterministic utility function u s.t. $u_{x}$ is integrable near to $x=0$, define $U(t, x)=\int_{0}^{x} Z_{t}\left(u_{x}(z)\right) d z$. Then

(i) $U(t, x)$ is an Itô semimartingale with local characteristics $\beta(t, x)=\int_{0}^{x} \mu\left(t, Z_{t}\left(u_{x}(z)\right) d z\right.$ and $\gamma(t, x)=\int_{0}^{x} \sigma\left(t, Z_{t}\left(u_{x}(z)\right) d z\right.$.

(ii) $U$ is a progressive utility with derivative $U_{x}(t, x)=Z_{t}\left(u_{x}(x)\right)$. Moreover, $U$ is a $\mathcal{K}_{\text {loc }}^{2, \varepsilon}$ semimartingale for any $0<\varepsilon<\delta$, with local characteristics $(\beta, \gamma)$ are of class $\mathcal{K}_{\text {loc }}^{2, \varepsilon} \times \overline{\mathcal{K}}_{\text {loc }}^{2, \varepsilon}$ for any $\varepsilon<\delta$.

(iii) If the $\operatorname{SDE}(\mu, \sigma)$ is of class $\mathcal{S}^{m, \delta},(m \geq 3, \delta \in(0,1])$, then, the progressive convex conjugate utility $\widetilde{U}$ of $U$ is a $\mathcal{K}_{l o c}^{m-1, \varepsilon}$-semimartingale.

- Its derivative $\widetilde{U}_{y}=-\left(U_{x}\right)^{-1}$ is a $\mathcal{K}_{l o c}^{m-2, \varepsilon}$-semimartingale, solution of the $\operatorname{SDE}(\tilde{\sigma}, \tilde{\mu})$ in $\mathcal{S}^{m, \varepsilon}$ for any $0<\varepsilon<\delta$, where

$$
\tilde{\sigma}(t,-z)=\frac{\gamma_{x}(t, z)}{U_{x x}(t, z)}, \quad \tilde{\mu}(t,-z)=\frac{1}{U_{x x}(t, z)}\left(\beta_{x}(t, z)-\frac{1}{2} \partial_{x}\left(\frac{\left\|\gamma_{x}(t, z)\right\|^{2}}{U_{x x}(t, z)}\right)\right)
$$

- Assumptions of Theorem 1.5 are satisfied and the dynamics of $\widetilde{U}$ and $\widetilde{U}_{y}$ are,

$$
\begin{aligned}
d \widetilde{U}(t, y) & \left.=\gamma\left(t,-\widetilde{U}_{y}(t, y)\right) \cdot d W_{t}+\beta\left(t,-\widetilde{U}_{y}(t, y)\right) d t+\frac{1}{2} \widetilde{U}_{y y}(t, y) \| \sigma_{t}(y)\right) \|^{2} d t \\
d \widetilde{U}_{y}(t, y) & \left.=-\widetilde{U}_{y y}(t, y) \sigma(t, y) \cdot d W_{t}+\left(\frac{1}{2} \partial_{y}\left(\|\sigma\|^{2} \partial_{y}\left(\widetilde{U}_{y}\right)\right)(t, y)-\mu(t, y) \partial_{y}\left(\widetilde{U}_{y}\right)(t, y)\right)\right) d t
\end{aligned}
$$

Concluding remarks on these two sections Having introduced the progressive utilities and their convex conjugate in Section 1, we have studied in detail conditions ensuring concavity and Inada conditions, by using that $U_{x}$ is a monotonic solution of a "regular" SDE. But the main 
result given in Section 2 concerns the inverse flow $\xi$ of the monotonic solution $X$ of a SDE, and the sufficient conditions on the coefficients ensuring the semimartingale property of the inverse flow $\xi$. The introduction of the adjoint SPDE satisfied by $\xi$ and the result of Theorem 2.7 are a powerful tool for that follows. We turn now to the study of additional condition called consistency property that we introduce in the next section.

\section{Consistent Dynamic Utilities}

\subsection{Definition of Consistent Dynamic Utilities}

The notion of progressive utility is very general and should be specified so as to represent more realistically the dynamic evolution of the individual preferences of an investor in a given financial market. The utility input provides a differential constraint on the risk attitude of the investor in terms of preference for higher or lower wealth, through the local risk tolerance $\tau^{U}(x, t)=-\frac{U_{x}(x, t)}{U_{x x}(x, t)}$, and, that we call, utility market risk premium $\eta^{U}(t, x)=\frac{\gamma_{x}(t, x)}{U_{x}(x, t)}$, revealing the interplay between the investment universe and the risk attitude.

The market input is described by a vector space $\mathscr{X}$ of portfolios incorporating feasibility and trading constraints and high liquidity. Several interpretations of the subclass of admissible portfolios can be done. The first one, proposed by Musiela and Zariphopoulou is that this class describes all investment universe. The second one is as follows: the market inputs may be viewed as a calibration universe, and the class $\mathscr{X}$ as a test-class of processes. The existence of an admissible portfolio giving the maximal satisfaction to the investor, which will be preserved at all times in the future, explains the martingale property in the definition below. On the other hand if the strategy in $\mathscr{X}$ fails to be optimal then it is better not to make investment. The optimal portfolio may be viewed as a benchmark for the investor using the utility $U$. Once his consistent progressive utility is defined, an investor can then turn to a portfolio optimization problem in a larger financial market or to calculate indifference prices. Following [25, 29], a $\mathscr{X}$-consistent dynamic utility is defined as follows.

Definition 3.1 ( $\mathscr{X}$-consistent dynamic utility). $A \mathscr{X}$-consistent dynamic utility $\mathbf{U}=\{U(t, x) ; t \geq$ $0, x>0\}$ is a progressive utility with the following additional properties:

CONSISTENCY with the test-ClAss: For any admissible wealth process $X \in \mathscr{X}$,

$$
\mathbb{E}\left(U\left(t, X_{t}\right) / \mathcal{F}_{s}\right) \leq U\left(s, X_{s}\right), \quad \forall s \leq t \text { a.s. }
$$

Existence OF OPTIMAL WeAlth: For any initial wealth $x>0$, there exists an optimal wealth process $X^{*} \in \mathscr{X}$ such that $X_{0}^{*}=x$, and for all $s \leq t$,

$$
U\left(s, X_{s}^{*}\right)=\mathbb{E}\left(U\left(t, X_{t}^{*}\right) / \mathcal{F}_{s}\right) \forall s \leq t \text { a.s. }
$$

In short for any admissible wealth $X \in \mathscr{X}, U(., X$.) is a positive supermartingale and a martingale for the optimal-benchmark wealth $X^{*}$.

Remark 3.1. (i) The martingale property can be weakened by the following localization procedure, if there exists a sequence of increasing stopping times $T_{n}\left(X_{0}\right) \nearrow \infty$ on the random interval 
$\left[0, T_{n}\left(X_{0}\right)\right], U\left(., X_{.}^{*}\right)$ is a martingale.

(ii) Note that the initial condition $U(0,)=.u($.$) is part of the definition, in particular u$ is a deterministic utility function which is fixed a priori independently of the given financial market and in particular of $\mathscr{X}$.

(iii) So a $\mathscr{X}$-consistent dynamic utility is a constant in time deterministic function only when the test-portfolios are local martingales. In this case, the optimal strategy is to do nothing.

(iv) Our definition differs slightly from the original $([25])$ since we do not require that the wealth processes $X$ are discounted. This variation offers more options and allows us to study the invariance of the class of dynamic utilities by change of numeraire. In any case, there is no fixed horizon.

\subsection{The investment universe}

We consider a incomplete Itô market, equipped with a $n$-standard Brownian motion, $W$ with Brownian coordinates $\left(W_{1}, W_{2}, \ldots, W_{n}\right)^{T}(n \geq d)$ and characterized by a short rate $\left(r_{t}\right)$ and a $n$-dimensional risk premium vector $(\eta)$. All these processes are defined on the filtered probability space $\left(\Omega, \mathcal{F}_{t \geq 0}, \mathbb{P}\right)$ satisfying usual assumptions, with minimal integrability assumptions. Since we only need to know the class of admissible portfolios, we immediately give the mathematical definition of this class, based on the self-financing equation without arbitrage. The market incompleteness is modeled as in Lehoczky, Karatzas, Shreve \& Xu [16]. The notations are the same than in Karatzas and Shreve [17] where the interested reader may be find all complementary information.

Definition 3.2 (Test portfolios). (i) A positive Itô semimartingale $X^{\kappa}$ is called a portfolio, or admissible wealth process if

$$
d X_{t}^{\kappa}=X_{t}^{\kappa}\left[r_{t} d t+\kappa_{t} .\left(d W_{t}+\eta_{t} d t\right)\right], \kappa_{t} \in \mathcal{R}_{t} .
$$

where $\kappa$ is a n-dimensional vector, progressive such that $\int_{0}^{T}\left\|\kappa_{t}\right\|^{2} d t<\infty$, a.s., measuring the volatility vector of the wealth $X^{\kappa}$.

(ii) The family of admissible wealth processes, also called test portfolios is defined by some restrictions on the volatility vector $\kappa$; we assume there exists progressive family of vector spaces $\left(\mathcal{R}_{t}\right)$ such that for any $t, \kappa_{t} \in \mathcal{R}_{t}$.

(iii) The family of test portfolios is denoted by $\mathscr{X}$. It may be easy to verify that $\mathscr{X}$ is convex.

The following short notations will be used extensively. Let $\mathcal{R}$ be a vector subspace of $\mathbb{R}^{n}$. For any $x \in \mathbb{R}^{n}, x^{\mathcal{R}}$ is the orthogonal projection of the vector $x$ onto $\mathcal{R}$ and $x^{\perp}$ is the orthogonal projection onto $\mathcal{R}^{\perp}$.

The existence of a risk premium $\eta$ is a possible formulation of the absence of arbitrage opportunity. Since from (3.1), the impact of the risk premium on the wealth dynamics only appears through the term $\kappa_{t} . \eta_{t}$ for $\kappa_{t} \in \mathcal{R}_{t}$, there is a "minimal" risk premium $\left(\eta_{t}^{\mathcal{R}}\right)$, the projection of $\eta_{t}$ on the space $\mathcal{R}_{t}\left(\kappa_{t} \cdot \eta_{t}=\kappa_{t} \cdot \eta_{t}^{\mathcal{R}}\right)$, to which we refer in the sequel. Moreover, the existence of $\eta^{\mathcal{R}}$ is not enough to insure the existence of equivalent martingale measure, since in general we do not know if the exponential local martingale $L_{t}^{\eta^{\mathcal{R}}}=\exp \left(\int_{0}^{t}-\eta_{s}^{\mathcal{R}} \cdot d W_{s}-\frac{1}{2} \int_{0}^{t}\left|\eta_{s}^{\mathcal{R}}\right|^{2} d s\right)$ is a uniformly integrable martingale, density of an equivalent martingale measure. Nevertheless, we are interested into the 
class of the so-called state price density processes $Y^{\nu}$ (taking into account the discount factor) who will play the same role for the dynamic conjugate utility, than the wealth processes $X^{\kappa}$ for the dynamic utility $U$.

Definition 3.3 (State price density process). (i) A Itô semimartingale $Y^{\nu}$ is called a state price density process if for any wealth process $X^{\kappa}, \kappa \in \mathcal{R}, Y^{\nu} X^{\kappa}$ is a local martingale. It follows that $Y^{\nu}$ satisfies,

$$
d Y_{t}^{\nu}=Y_{t}^{\nu}\left[-r_{t} d t+\left(\nu_{t}-\eta_{t}^{\mathcal{R}}\right) \cdot d W_{t}\right], \quad \nu_{t} \in \mathcal{R}_{t}^{\perp}, \quad Y_{0}^{\nu}=y
$$

(ii) Denote $\mathscr{Y}$ the convex family of all state density processes $Y^{\nu}$ where $\nu \in \mathcal{R}^{\perp}$ and observe that $Y^{\nu}$ is the product of $Y^{0}(\nu=0)$ by the density martingale $L_{t}^{\nu}=\exp \left(\int_{0}^{t} \nu_{s} \cdot d W_{s}-1 / 2 \int_{0}^{t}\left|\nu_{s}\right|^{2} d s\right)$.

\subsection{Consistent Dynamic Utility and Portfolio Optimization}

$\mathscr{X}$-consistent dynamic utility and HJB constraint In Paragraph 1.2 more precisely in Theorem 1.1, we have characterized progressive utilities in terms of their local characteristics $(\beta, \gamma)$ as well as in terms of the parameters $(\mu, \sigma)$ of the intrinsic SDE (1.3) satisfied by $U_{x}$. In this section, we are concerned with the constraint induced on the drift characteristic $\beta$ of the dynamic utility by the consistency property. The consistency property plays the same role that the dynamic programming principle in the classical theory of backward expected utility maximization, (see for example H. Pham [30]). Thanks to Itô-Ventzel's formula (Theorem [1.4), constraints on the local characteristics $(\beta, \gamma)$ of $\mathbf{U}$ lead to non standard Hamilton-Jacobi-Bellman Stochastic PDE. As in the classical case, the main parameters of the SPDE are the risk tolerance process and the utility market risk premium.

Definition 3.4 (Utility risk tolerance and risk premium). In this financial framework, the utility risk tolerance random field is defined by $\tau^{U}(t, x)=-\frac{U_{x}(t, x)}{U_{x x}(t, x)}$ and the utility risk premium random field by $\eta^{U}(t, x)=\frac{\gamma_{x}(t, x)}{U_{x}(t, x)}$ with its two components $\eta^{U, \mathcal{R}} \in \mathcal{R}, \eta^{U, \perp} \in \mathcal{R}^{\perp}$.

Observe that Condition (1.8) in Corollary 1.3 states that $\eta^{U}$ is bounded in $x$ with random bound.

The supermartingale property of $U\left(., X^{\kappa}\right)$ implies that the drift of these processes must be negative for all $\kappa \in \mathcal{R}$, and equal to 0 for some $\kappa^{*}$. We proceed by verification as in the classical case.

Theorem 3.1 (Utility-SPDE). Let $\mathbf{U}$ be a progressive utility which is a $\mathcal{K}_{\text {loc }}^{2, \delta}$-semimartingale $(\delta \in(0,1])$ with local characteristics $(\beta, \gamma)$. Assume the drift constraint to be of HJB type,

$$
\beta(t, x)=-U_{x}(t, x) r_{t} x-\frac{1}{2} U_{x x}(t, x) \inf _{\kappa \in \mathcal{R}}\left\{\|x \kappa\|^{2}+2 x \kappa \cdot\left(\frac{U_{x}(t, x) \eta_{t}^{\mathcal{R}}+\gamma_{x}(t, x)}{U_{x x}(t, x)}\right)\right\} .
$$

(i) The minimum of the quadratic form (3.3) is achieved at the optimal policy $\kappa^{*}$ given by

$$
\left\{\begin{array}{cl}
x \kappa_{t}^{*}(x) & =-\frac{1}{U_{x x}(t, x)}\left(U_{x}(t, x) \eta_{t}^{\mathcal{R}}+\gamma_{x}^{\mathcal{R}}(t, x)\right) \\
\text { and } \beta(t, x) & =-U_{x}(t, x) x r_{t}+\frac{1}{2} U_{x x}(t, x)\left\|x \kappa^{*}(t, x)\right\|^{2}
\end{array}\right.
$$

(ii) For any $\kappa \in \mathcal{R}$, the process $U\left(., X_{.}^{\kappa}\right)$ is a supermartingale, and a local martingale for any solution (if there exists) $X^{*}$ of the $S D E d X_{t}^{*}=X_{t}^{*}\left(r_{t} d t+\kappa^{*}\left(t, X_{t}^{*}\right) \cdot\left(d W_{t}+\eta_{t}^{\sigma} d t\right)\right)$. Under additional integrability assumptions, the $\mathscr{X}$-consistency property is satisfied. 
Remark 3.2. In the classical backward framework, similar SPDE is investigated by Mania and Tevzadze 22] using BSDE tools, and by Englezios and Karatzas [14]. (See also Remark 4.2)

Proof. By Itô-Ventzel's formula (Theorem 1.4), for any admissible portfolio $X^{\kappa}$,

$$
\begin{aligned}
d U\left(t, X_{t}^{\kappa}\right) & =\left(U_{x}\left(t, X_{t}^{\kappa}\right) X_{t}^{\kappa} \kappa_{t}+\gamma\left(t, X_{t}^{\kappa}\right)\right) \cdot d W_{t} \\
& +\left(\beta\left(t, X_{t}^{\kappa}\right)+U_{x}\left(t, X_{t}^{\kappa}\right) r_{t} X_{t}^{\kappa}+\frac{1}{2} U_{x x}\left(t, X_{t}^{\kappa}\right) \mathcal{Q}\left(t, X_{t}^{\kappa}, \kappa_{t}\right)\right) d t, \\
\text { where } \mathcal{Q}(t, x, \kappa) & :=\|x \kappa\|^{2}+2 x \kappa \cdot\left(\frac{U_{x}(t, x) \eta_{t}^{\mathcal{R}}+\gamma_{x}(t, x)}{U_{x x}(t, x)}\right) .
\end{aligned}
$$

Since $\kappa \in \mathcal{R}, \mathcal{Q}(t, x, \kappa)$ is only depending on $\gamma_{x}^{\mathcal{R}}(t, x)$, the orthogonal projection of $\gamma_{x}(t, x)$ on $\mathcal{R}_{t}$. The minimum $\mathcal{Q}^{*}(t, x)=\inf _{\kappa \in \mathcal{R}} \mathcal{Q}(t, x, \kappa)$ of the quadratic form $\mathcal{Q}(t, x, \kappa)$ is achieved at the optimal policy $\kappa^{*}$ given by

$$
\left\{\begin{array}{l}
x \kappa_{t}^{*}(x)=-\frac{1}{U_{x x}(t, x)}\left(U_{x}(t, x) \eta_{t}^{\mathcal{R}}+\gamma_{x}^{\mathcal{R}}(t, x)\right) \\
\left.\mathcal{Q}^{*}(t, x)=-\frac{1}{U_{x x}(t, x)^{2}} \| U_{x}(t, x) \eta_{t}^{\mathcal{R}}+\gamma_{x}^{\mathcal{R}}(t, x)\right)\left\|^{2}=-\right\| x \kappa_{t}^{*}(x) \|^{2} .
\end{array}\right.
$$

Then the drift of the semimartingale $U\left(t, X_{t}^{\kappa}\right)$ satisfies

$$
\begin{aligned}
& \beta\left(t, X_{t}^{\kappa}\right)+U_{x}\left(t, X_{t}^{\kappa}\right) r_{t} X_{t}^{\kappa}+\frac{1}{2} U_{x x}\left(t, X_{t}^{\kappa}\right) \mathcal{Q}\left(t, X_{t}^{\kappa}, \kappa_{t}\right) \\
\leq & \beta\left(t, X_{t}^{\kappa}\right)+U_{x}\left(t, X_{t}^{\kappa}\right) r_{t} X_{t}^{\kappa}+\frac{1}{2} U_{x x}\left(t, X_{t}^{\kappa}\right) \mathcal{Q}^{*}\left(t, X_{t}^{\kappa}, \kappa_{t}\right) \\
= & \beta\left(t, X_{t}^{\kappa}\right)+U_{x}\left(t, X_{t}^{\kappa}\right) r_{t} X_{t}^{\kappa}-\frac{1}{2} U_{x x}\left(t, X_{t}^{\kappa}\right)\left\|X_{t}^{\kappa} \kappa_{t}^{*}\left(X_{t}^{\kappa}\right)\right\|^{2}
\end{aligned}
$$

The proof is complete.

Conjugate of consistent dynamic utility The characteristics of the conjugate progressive utility $\widetilde{\mathbf{U}}$ can be computed directly from Theorem 1.5. Given that $\beta$ is associated with an optimization program, we show that the dual drift $\tilde{\beta}$ is also constrained by a HJB type relation in the new variables. So, the convex conjugate utility $\widetilde{\mathbf{U}}$ is consistent with a family of state price density processes (Definition 3.3). As observed in Theorem 2.8 (ii), the study of the conjugate utility $\widetilde{U}$ requires stronger assumptions than the study of $U$.

Theorem 3.2. Let $\mathbf{U}$ a progressive utility with characteristics $(\beta, \gamma)$ satisfying Assumptions of Theorem [2.8. Then its progressive convex conjugate utility $\widetilde{\mathbf{U}}$ and its marginal conjugate utility $\widetilde{\mathbf{U}}_{\mathbf{y}}$ are Itô random fields with local characteristics $(\tilde{\beta}, \tilde{\gamma})$ and $\left(\tilde{\beta}_{y}, \tilde{\gamma}_{y}\right)$ respectively. Assume the drift constraint of $\mathbf{U}$ to be of HJB type (3.3).

(i) The local characteristics of the convex conjugate $\widetilde{\mathbf{U}}$ are given by:

$$
\left\{\begin{array}{l}
\tilde{\gamma}(t, y):=\gamma\left(t,-\widetilde{U}_{y}(t, y)\right), \quad \tilde{\gamma}_{y}(t, y):=-\gamma_{x}\left(t,-\widetilde{U}_{y}(t, y)\right) \cdot \widetilde{U}_{y y}(y) \\
\tilde{\beta}(t, y)=y \widetilde{U}_{y}(t, y) r_{t}+\frac{1}{2 \widetilde{U}_{y y}(t, y)}\left(\left\|\tilde{\gamma}_{y}(t, y)\right\|^{2}-\left\|\tilde{\gamma}_{y}^{\mathcal{R}}(t, y)+y \widetilde{U}_{y y}(t, y) \eta_{t}^{\mathcal{R}}\right\|^{2}\right)
\end{array}\right.
$$

(ii) The non linear drift $\tilde{\beta}(t, y)$ is associated with the following optimization program:

$$
\tilde{\beta}(t, y)=y \widetilde{U}_{y}(t, y) r_{t}-\frac{1}{2} y^{2} \widetilde{U}_{y y}(t, y) \inf _{\nu_{t} \in \mathcal{R}^{\perp}}\left\{\left\|\nu_{t}-\eta_{t}^{\mathcal{R}}\right\|^{2}+2\left(\nu_{t}-\eta_{t}^{\mathcal{R}}\right) \cdot\left(\frac{\tilde{\gamma}_{y}(t, y)}{y \widetilde{U}_{y y}(t, y)}\right)\right\}
$$


(iii) The minimum of this quadratic form is achieved at the optimal policy

$$
y \nu_{t}^{*}(y)=\frac{-\tilde{\gamma}_{y}^{\perp}(t, y)}{\widetilde{U}_{y y}(t, y)}=\gamma_{x}^{\perp}\left(t,-\widetilde{U}_{y}(t, y)\right)=y \eta^{U, \perp}\left(t,-\widetilde{U}_{y}(t, y)\right) .
$$

(iv) Rewritten in terms of optimal strategy, $\tilde{\beta}(t, y)$ becomes

$$
\tilde{\beta}(t, y)=y \widetilde{U}_{y}(t, y) r_{t}-\frac{1}{2} \widetilde{U}_{y y}(t, y)\left[\left\|y \nu_{t}^{*}(y)-y \eta_{t}^{\mathcal{R}}\right\|^{2}-2\left(y \nu_{t}^{*}(y)-y \eta_{t}^{\mathcal{R}}\right) \cdot \frac{\tilde{\gamma}_{y}(t, y)}{\widetilde{U}_{y y}(t, y)}\right] .
$$

(v)For any admissible state price density process $Y^{\nu} \in \mathscr{Y}$ with $\nu \in \mathcal{R}^{\perp}, \widetilde{U}\left(t, Y_{t}^{\nu}\right)$ is a submartingale, and a local martingale for any solution $Y^{*}$ (if there exists) of the equation $d Y_{t}^{*}=Y_{t}^{*}\left[-r_{t} d t+\right.$ $\left.\left(\nu^{*}\left(t, Y_{t}^{*}\right)-\eta_{t}^{\mathcal{R}}\right) \cdot d W_{t}\right]$.

Proof. (i) By Theorem 1.5 the local characteristics $(\tilde{\beta}, \tilde{\gamma})$ of the conjugate random field $\widetilde{U}$ are given by $\tilde{\gamma}(t, y)=\gamma(t,-\widetilde{U}(t, y))$ and $\tilde{\beta}(t, y)=\beta^{1}(t,-\widetilde{U}(t, y))$ where $\beta^{1}(t, x)=\beta(t, x)-$ $\frac{1}{2 U_{x x}(t, x)}\left\|\gamma_{x}(t, x)\right\|^{2}$. Combining this identity with the HJB-constraint $\beta(t, x)=-U_{x}(t, x) x r_{t}+$ $\frac{1}{2} U_{x x}(t, x)\left\|x \kappa^{*}(t, x)\right\|^{2}$ yields to

$$
\beta^{1}(t, x)=-x U_{x}(t, x) r_{t}-\frac{1}{2 U_{x x}(t, x)}\left(\left\|\gamma_{x}(t, x)\right\|^{2}-\left\|U_{x}(t, x) \eta_{t}^{\mathcal{R}}+\gamma_{x}^{\mathcal{R}}(t, x)\right\|^{2}\right) .
$$

(ii) \& (iii) Since the norm of the projection on $\mathcal{R}_{t}$ is the distance to the orthogonal vector space $\mathcal{R}_{t}^{\perp}$

$$
\left\|\frac{\gamma_{x}^{\mathcal{R}}(t, x)}{U_{x}(t, x)}+\eta_{t}^{\mathcal{R}}\right\|^{2}:=\left\|\eta^{U, \mathcal{R}}(t, x)+\eta_{t}^{\mathcal{R}}\right\|^{2}=\inf _{\nu \in \mathcal{R}_{t}^{\perp}}\left\|\nu-\left(\eta^{U, \mathcal{R}}+\eta_{t}^{\mathcal{R}}\right)\right\|^{2}
$$

Using the relation $|x|^{2}-|y|^{2}=|x-y|^{2}+2(x-y)$. $y$, we get:

$$
\begin{aligned}
\left\|\eta^{U, \mathcal{R}}(t, x)+\eta_{t}^{\mathcal{R}}\right\|^{2}-\left\|\eta^{U}(t, x)\right\|^{2} & =\inf _{\nu \in \mathcal{R} \perp}\left\{\left\|\nu-\eta_{t}^{\mathcal{R}}\right\|^{2}+2\left(\nu-\eta_{t}^{\mathcal{R}}\right) \cdot \eta^{U}(t, x)\right\} \\
& =\left\|\eta^{U, \perp}(t, x)-\eta_{t}^{\mathcal{R}}\right\|^{2}+2\left(\eta^{U, \perp}(t, x)-\eta_{t}^{\mathcal{R}}\right) \cdot \eta^{U}(t, x) .
\end{aligned}
$$

By coming back to $\beta^{1}$, we can make the minimization program to be explicit,

$$
\begin{aligned}
\beta^{1}(t, x)+x U_{x}(t, x) r_{t} & =\frac{U_{x}^{2}(t, x)}{\left.U_{x x} t, x\right)}\left(\inf _{\nu \in \mathcal{R}^{\perp}}\left\{\left\|\nu-\eta_{t}^{\mathcal{R}}\right\|^{2}+2\left(\nu-\eta_{t}^{\mathcal{R}}\right) \cdot \eta^{U}(t, x)\right\}\right) \\
& =\frac{U_{x}^{2}(t, x)}{\left.U_{x x} t, x\right)}\left(\left\|\eta^{U, \perp}(t, x)-\eta_{t}^{\mathcal{R}}\right\|^{2}+2\left(\eta^{U, \perp}(t, x)-\eta_{t}^{\mathcal{R}}\right) \cdot \eta^{U}(t, x)\right) .
\end{aligned}
$$

The minimum in the quadratic form is achieved at $\eta^{U, \perp}(t, x)$, corresponding the optimal strategy $\nu_{t}^{*}(y)=\eta^{U, \perp}\left(t,-\widetilde{U}_{y}(t, y)\right)$. From this and the identities,

$$
\frac{U_{x}^{2}\left(t,-\widetilde{U}_{y}(t, y)\right)}{2 U_{x x}\left(t,-\widetilde{U}_{y}(t, y)\right)}=-\frac{1}{2} y^{2} \widetilde{U}_{y y}(t, y), \quad \eta^{U}\left(t,-\widetilde{U}_{y}(t, y)\right)=-\frac{\tilde{\gamma}_{y}(t, y)}{y \widetilde{U}_{y y}(t, y)}
$$

we get the desired formula for $\tilde{\beta}$ both in (3.6) and in (3.8), i.e.

$$
\begin{aligned}
\tilde{\beta}(t, y) & =y \widetilde{U}_{y}(t, y) r_{t}+\frac{1}{2 \widetilde{U}_{y y}(t, y)}\left(\left\|\tilde{\gamma}_{y}(t, y)\right\|^{2}-\left\|\tilde{\gamma}_{y}^{\mathcal{R}}(t, y)+y \widetilde{U}_{y y}(t, y) \eta_{t}^{\mathcal{R}}\right\|^{2}\right) \\
& =y \widetilde{U}_{y}(t, y) r_{t}-\frac{1}{2} \widetilde{U}_{y y}(t, y)\left[\left\|y \nu_{t}^{*}(y)-y \eta_{t}^{\mathcal{R}}\right\|^{2}-2\left(y \nu_{t}^{*}(y)-y \eta_{t}^{\mathcal{R}}\right) \cdot \frac{\tilde{\gamma}_{y}(t, y)}{\widetilde{U}_{y y}(t, y)}\right]
\end{aligned}
$$

(iv) is a simple rewriting of the HJB constraint on $\tilde{\beta}$.

(v) The supermartingale property is proved in very similar manner than for dynamic utility, (proof of Proposition 3.2 (ii) and (iii)). 


\section{Marginal Utility SPDE and Optimal SDEs}

As seen in Section 1 and 2, our utility characterization is based on the marginal utility. So, we focus now on the dynamics of the random field $\mathbf{U}_{\mathbf{x}}$. As established in Theorem 3.1, the drift characteristic of $\mathcal{X}$-consistent utility is constraint by a HJB type condition (3.3)

$$
\beta(t, x)=-U_{x}(t, x) x r_{t}+\frac{1}{2} U_{x x}(t, x)\left\|x \kappa_{t}^{*}(x)\right\|^{2} .
$$

The diffusion characteristic $\gamma_{x}$ is explained by the optimal policies of the primal and dual problems, Theorem 3.1 and Theorem 3.2

$$
\gamma_{x}(t, x)=-U_{x x}(t, x) x \kappa_{t}^{*}(x)-U_{x} \eta_{t}^{\mathcal{R}}+U_{x}(t, x) \nu_{t}^{*}\left(U_{x}(t, x)\right)
$$

The characteristic $\gamma_{x}(t, x)$ may be rewritten in a more convenient form, using the diffusion coefficient of the optimal policy $\sigma_{t}^{*}(x):=x \kappa_{t}^{*}(x)$, and the diffusion coefficient of the optimal state price density $\tilde{\sigma}_{t}^{*}(y)=-y \eta_{t}^{\mathcal{R}}+\gamma_{x}^{\perp}\left(t,-\widetilde{U}_{y}(t, y)\right)$ as,

$$
\gamma_{x}(t, x)=-\partial_{x}\left(U_{x}\right)(t, x) \sigma_{t}^{*}(x)-U_{x}(t, x) \eta_{t}^{\mathcal{R}}+\tilde{\sigma}_{t}^{*}\left(U_{x}(t, x)\right)
$$

It is easy to recognize the diffusion coefficient of the SPDE (2.9) in Theorem 2.7 associated with the SDEs with diffusion parameters $\sigma_{t}^{*}(x)$ and $\tilde{\sigma}_{t}^{*}(y)$. Moreover, by taking the $x$-derivative in the drift constraint $\beta(t, x)=-U_{x}(t, x) x r_{t}+\frac{1}{2} U_{x x}(t, x)\left\|x \kappa_{t}^{*}(x)\right\|^{2}=-U_{x}(t, x) x r_{t}+\frac{1}{2} U_{x x}(t, x)\left\|\sigma_{t}^{*}(x)\right\|^{2}$, it appears naturally a divergence term associated with the optimal policy $\sigma_{t}^{*}(x)=x \kappa_{t}^{*}(x)$ in the drift characteristic of $\mathbf{U}_{\mathbf{x}}$ which suggests to use the main theorem (Theorem 2.7) applied to the optimal SDEs.

\subsection{Main result : solving marginal utility SPDE via optimal SDEs}

To be closer to the notation of Theorem 2.7. we recall all the coefficients of SDEs associated with the optimal policies $X^{*}$ and $Y^{*}$ if they exist:

$$
\begin{cases}\mu_{t}^{*}(x) & :=r_{t} x+x \kappa_{t}^{*}(x) \cdot \eta_{t}^{\mathcal{R}}, \quad \sigma_{t}^{*}(x):=x \kappa_{t}^{*}(x) \\ \tilde{\mu}_{t}^{*}(y) & :=-r_{t} y, \\ \widehat{L}_{t, x}^{*} & :=\frac{1}{2} \partial_{x}\left(\left\|\sigma_{t}^{*}(x)\right\|^{2} \partial_{x}\right)-\mu_{t}^{*}(x) \partial_{x}\end{cases}
$$

We start with the identification of the SPDE satisfied by the marginal utility of a consistent dynamic utility imposing only regularity condition on the utility random field and its local characteristics. We will then give additional conditions that guarantee the existence of solution to SDEs with coefficients $\left(\mu^{*}, \sigma^{*}\right)$ and $\left(\tilde{\mu}^{*}, \tilde{\sigma}^{*}\right)$.

Proposition 4.1. Let $U$ be a $\mathcal{K}_{\text {loc }}^{2, \delta} \cap \mathcal{C}^{3}$-regular $(\delta>0)$ progressive utility $U$, whose the local characteristics $(\beta, \gamma)$ satisfy the HJB constraints,

$$
\begin{cases}\gamma_{x}(t, x) & :=-U_{x x}(t, x) \sigma_{t}^{*}(x)+\eta_{t}^{\mathcal{R}} U_{x}(t, x)+\tilde{\sigma}_{t}^{*}\left(U_{x}(t, x)\right) \\ \beta(t, x) & :=-U_{x}(t, x) x r_{t}+\frac{1}{2} U_{x x}(t, x)\left\|\sigma_{t}^{*}(x)\right\|^{2}\end{cases}
$$

The marginal utility $\mathbf{U}_{\mathbf{x}}$ is a decreasing solution of the SPDE(2.9) with coefficients $\left(\mu^{*}, \sigma^{*}\right)$ and $\left(\tilde{\mu}^{*}, \tilde{\sigma}^{*}\right)$

$$
\begin{aligned}
d U_{x}(t, x) & =\tilde{\sigma}_{t}^{*}\left(U_{x}(t, x)\right) \cdot d W_{t}+\tilde{\mu}_{t}^{*}\left(U_{x}(t, x)\right) d t \\
& -\partial_{x} U_{x}(t, x) \sigma_{t}^{*}(x) \cdot\left(d W_{t}+\tilde{\sigma}_{y}^{*}\left(t, U_{x}(t, x)\right) d t\right)+\widehat{L}_{t, x}^{*}\left(U_{x}\right) d t
\end{aligned}
$$


Proof. At first, as $U$ is assumed to be $\mathcal{K}_{\text {loc }}^{2, \delta} \cap \mathcal{C}^{(3)}$-regular, $U_{x}$ is of class $\mathcal{K}_{\text {loc }}^{1, \delta}$ and its local characteristics $\left(\beta_{x}, \gamma_{x}\right)$ are of class $\mathcal{C}^{1}$ in $x$; then, the vectors $\sigma_{t}^{*}(x)=x \kappa_{t}^{*}(x)=-\left(\gamma_{x}^{\mathcal{R}}(t, x)+\right.$ $\left.\eta_{t}^{\mathcal{R}} U_{x}(t, x)\right) / U_{x x}(t, x)$ and $\tilde{\sigma}_{t}^{*}(y)=-\eta_{t}^{\mathcal{R}} y+\gamma_{x}^{\perp}\left(t,-\widetilde{U}_{y}(t, y)\right)$ are also of class $\mathcal{C}^{1}$, necessary condition to define $\widehat{L}^{*}$. By derivation of the local characteristics, it is clear that $\beta_{x}$ contains a second order term in divergence form associated with $x \kappa_{t}^{*}(x)=\sigma_{t}^{*}(x)$, which leads us to introduce the adjoint operator $\widehat{L}_{t, x}^{*}$ associated with $\left(\mu^{*}, \sigma^{*}\right)$. It remains to make some slight transformations on the drift characteristic. Observe that

$$
\begin{aligned}
\beta_{x}(t, x) & =-\partial_{x}\left(U_{x}(t, x) x r_{t}\right)+\partial_{x}\left(\frac{1}{2} U_{x x}(t, x)\left\|\sigma_{t}^{*}(x)\right\|^{2}\right) \\
& =\widehat{L}_{t, x}^{*}\left(U_{x}\right)-r_{t} U_{x}(t, x)+\partial_{x} U_{x}(t, x) \sigma_{t}^{*}(x) \cdot \eta_{t}^{\mathcal{R}} \\
& =\widehat{L}_{t, x}^{*}\left(U_{x}\right)+\tilde{\mu}_{t}^{*}\left(U_{x}\right)+\partial_{x} U_{x}(t, x) \sigma_{t}^{*}(x) \cdot \eta_{t}^{\mathcal{R}}
\end{aligned}
$$

Let us give another interpretation of $\sigma_{t}^{*}(x) \cdot \eta_{t}^{\mathcal{R}}$. Since $\tilde{\sigma}^{*}(t, y)+\eta_{t}^{\mathcal{R}} y$ belongs to the vector space $\mathcal{R}_{t}^{\perp}$ the spatial derivative $\tilde{\sigma}_{y}^{*}(t, y)+\eta_{t}^{\mathcal{R}}$ is also in $\mathcal{R}_{t}^{\perp}$, yielding to the relation on the scalar products $-\sigma_{t}^{*}(x) \cdot \eta_{t}^{\mathcal{R}}=\sigma_{t}^{*}(x) \cdot \tilde{\sigma}_{y}^{*}(t, y)$. Then, Identity (4.3) holds true.

Comment (i) There is a fairly subtle relation between the $\operatorname{SDE}\left(\mu^{U}, \sigma^{U}\right)$ introduced in Theorem 1.1 to characterize the marginal utility $\mathbf{U}_{\mathbf{x}}$ where $\mu^{U}(t, z)=\beta_{x}\left(t,-\widetilde{U}_{y}(t, z)\right)$ and $\sigma^{U}(t, z)=$ $\gamma_{x}^{U}\left(t,-\widetilde{U}_{y}(t, z)\right)$, and the coefficients of the two optimal SDEs, in particular in terms of diffusion coefficients. The HJB constraint $\left.\gamma_{x}^{U}(t, x)=-U_{x x}(t, x) \sigma_{t}^{*}(x)+\eta_{t}^{\mathcal{R}} U_{x}(t, x)\right)+\tilde{\sigma}_{t}^{*}\left(U_{x}(t, x)\right)$ becomes

$$
\sigma^{U}(t, z)=\frac{\sigma_{t}^{*}\left(-\widetilde{U}_{y}(t, z)\right)}{\widetilde{U}_{y y}(t, z)}+\eta_{t}^{\mathcal{R}} z+\tilde{\sigma}_{t}^{*}(z)
$$

In particular, since $\tilde{\sigma}_{t}^{*}(z)=\sigma^{U, \perp}(t, z)$, any regularity property on $\sigma^{U}$ are immediately transferred by linear projection on $\tilde{\sigma}_{t}^{*}(-z)$. But, only some local regularity on $\sigma_{t}^{*}(t, z)$ may be deduced from global regularity of $\sigma^{U}$. Nevertheless, we can justify the existence of a global optimal wealth solution in a similar way than for the inverse process.

(ii) Observe also that we obtained a way to generated $\mathcal{X}$-consistent utility only from their local characteristics and the SPDE 4.3 since we do not use a priori the concavity assumption in the following theorem.

Theorem 4.2 (Main theorem). Let $U$ be a $\mathcal{K}_{\text {loc }}^{2, \delta} \cap \mathcal{C}^{3}$-semimartingale progressive utility $U$, whose the local characteristics $(\beta, \gamma)$ satisfy the HJB constraints [4.2]) then, the derivative $U_{x}(t, x)$ is solution of the SPDE (4.3).

Assume, in addition, the existence of two positive adapted stochastic bounds $\left(K^{1}, K^{2}\right)$ such that

$$
\left\|\gamma_{x}^{\perp}(t, x)\right\| \leq K_{t}^{1}\left|U_{x}(t, x)\right|,\left\|\gamma_{x x}^{\perp}(t, x)\right\| \leq K_{t}^{2}\left|U_{x x}(t, x)\right|, \text { a.s., }\left(K^{1}, K^{2}\right) \in \mathcal{L}^{2}(d t)
$$

Existence of optimal PRocesses: (i) The conjuguate $S D E\left(\tilde{\mu}^{*}, \tilde{\sigma}^{*}\right)$ is uniformly Lipschitz and has a unique strong solution $Y_{t}^{*}(y)$, which is strictly positive, and strictly monotonic, with range $[0, \infty)$.

(ii) The $\operatorname{SDE}\left(\mu^{*}, \sigma^{*}\right)$ has only local Lipschitz property, and admits a maximal monotonic solution $X^{*}$ defined a priori only up to a stopping times $\zeta^{*}(x)$. But, the explosion time $\zeta^{*}(x)=\infty$ a.s. since the processes $U_{x}\left(., X^{*}(x)\right)$ is distinguishable from the solution $Y_{.}^{*}\left(u_{x}(x)\right)$.

CONSISTENCY AND MARGINAL UTILITY CHARACTERIZATION: The random field $U$ is a $\mathcal{X}$-consistent 
utility, with optimal wealth $X^{*}$. Furthermore, the derivative random field $U_{x}(t, x)$, solution of the SPDE (4.3) is given by $U_{x}(t, x)=Y_{t}^{*}\left(u_{x}\left(\left(X_{t}^{*}\right)^{-1}(x)\right)\right)$. So $U_{x}(t, x)$ is a strictly decreasing and positive process with range $[\infty, 0]$.

Remark 4.1. In this framework, we do not need that the inverse process $\mathcal{X}(t, x)$ is a semimartingale. Nevertheless, if $\gamma_{x}^{\perp} \equiv 0$, then $Y_{t}^{*}(y)=y Y_{t}^{0}$ and as $U_{x}(t, x)$ is a semimartingale, $u_{x}(\mathcal{X}(t, x))$ is also semimartingale, consequently $\mathcal{X}(., x)$ is a semimartingale since $U(t, x)$ and $u$ are assumed to be of class $\mathcal{C}^{3}$.

Proof. (i) The assumption (4.5) implies as above observed that the coefficients $\left(\tilde{\mu}^{*}, \tilde{\sigma}^{*}\right)$ are uniformly Lipschitz in space, and then the SDE has an unique monotonic strong solution $Y_{t}^{*}(y)$. Since by assumption $\left\|\gamma_{x}(t, x)\right\| \leq K_{t}^{1}\left|U_{x}(t, x)\right|$, then $\left\|\tilde{\alpha}^{*}(t, y)\right\|=\frac{\left\|\tilde{\sigma}^{*}(t, y)\right\|}{y} \leq K_{t}^{1}$. As $K^{1} \in \mathcal{L}^{2}(d t), Y^{*}$ which is also solution of the $\operatorname{SDE} d Y_{t}^{*}=Y_{t}^{*}\left[r_{t} d t+\tilde{\alpha}^{*}\left(t, Y_{t}^{*}\right) \cdot d W_{t}\right.$ is strictly positive.

(ii) Under the same assumption in addition to the property of $U$ of class $\mathcal{K}_{l o c}^{2, \delta}$, the coefficients $\left(\mu^{*}, \sigma^{*}\right)$ are only in $\left.\mathcal{K}_{l o c}^{0,1} \times \overline{\mathcal{K}}_{l o c}^{0,1}\right)$, and then by Theorem 2.4, there exists a monotonic maximal solution $X_{t}^{*}(x)$ up to explosion time $\zeta^{*}(x)$.

(iii) a) We show together the property $\left.\left(U_{x}\left(t, X_{t}^{*}(x)\right)=Y_{t}^{*}\left(u_{x}\right)\right)\right)$ and the fact that $\zeta^{*}(x)=\infty$. We are applying, up to $\zeta^{*}(x)$, Theorem 2.7 iv) to $U_{x}(t, x)$, solution of the SDPE (4.3), and to the process $X_{t}^{*}$. Then, it follows that $U_{x}\left(t, X_{t}^{*}(x)\right)$ is solution of $\operatorname{SDE}\left(\tilde{\mu}^{*}, \tilde{\sigma}^{*}\right)$ up to $\zeta^{*}(x)$ with initial condition $u_{x}$. In other words, $\left.U_{x}\left(t, X_{t}^{*}(x)\right)=Y_{t}^{*}\left(u_{x}(x)\right)\right) ; t<\zeta^{*}(x)$.

b) When $t \mapsto \zeta^{*}(x)$ on $\zeta^{*}(x)<\infty, U_{x}\left(t, X_{t}^{*}(x)\right) \mapsto 0$, and $\left.Y_{t}^{*}\left(u_{x}(x)\right)\right) \mapsto Y_{\zeta^{*}(x)}^{*}$, then $\left.Y_{\zeta^{*}(x)}^{*}\left(u_{x}(x)\right)\right)=$ 0 , but since $Y_{t}^{*}(y)$ is a strictly positive process, a contradiction occurs if $\mathbb{P}\left(\zeta^{*}(x)<\infty\right)>0$.

(iv) The property of $U$ are deduced of the identity $\left.U_{x}\left(t, X_{t}^{*}(x)\right)=Y_{t}^{*}\left(u_{x}(x)\right)\right)$ and from Theorem

\section{8}

Remark 4.2. In 14] the authors have also shown that the solution of the backward SPDE can be represented through the composition of two invertible processes. The main difference with the approach proposed here is that their processes are given as conditional expectation of monotonic functions as we describe them in path-wise form as SDE solutions.

Comment: Obviously the main result, Theorem 4.2, gives us only sufficient conditions to generate $\mathcal{X}$-consistent utility from their characteristics. In fact, the assumptions gives us the existence of a monotonic strictly positive strong solution $Y^{*}$ of $\operatorname{SDE}\left(\tilde{\mu}^{*}, \tilde{\sigma}^{*}\right)$, and by local regularity, the existence of a monotonic solution $X^{*}$ of $\operatorname{SDE}\left(\tilde{\mu}^{*}, \tilde{\sigma}^{*}\right)$ up to explosion time $\zeta^{x}$. Obviously, we can interchange the roles of $Y^{*}$ and $X^{*}$. Indeed, in line with Section 2, given the existence of a solution of the SPDE (4.3), by assertion (iii) of Theorem 2.7 the existence of a solution $X^{*}(x)$ starting from $x$ to the $\operatorname{SDE}\left(\mu^{*}, \sigma^{*}\right)$ implies that $U_{x}\left(t, X_{t}^{*}(x)\right)$ is solution of the $\operatorname{SDE}\left(\tilde{\mu}^{*}, \tilde{\sigma}^{*}\right)$ and so is a state price density process $\widehat{Y}_{t}^{*}\left(u_{x}(x)\right)$, starting from $u_{x}(x)$. Moreover, existence of a solution to $\operatorname{SDE}\left(\mu^{*}, \sigma^{*}\right)$ is equivalent to existence of a solution to $\operatorname{SDE}\left(\tilde{\mu}^{*}, \tilde{\sigma}^{*}\right)$. Otherwise, if we give ourselves $X^{*}$ monotone solution of $\operatorname{SDE}\left(\mu^{*}, \sigma^{*}\right)$ with a semimartingale inverse $\mathcal{X}$ and $Y^{*}$ a $\mathcal{K}^{2}$ regular solution to $\operatorname{SDE}\left(\tilde{\mu}^{*}, \tilde{\sigma}^{*}\right)$ then, from assertion $(i)$ of Theorem 2.7 the compound process $\widehat{Y}_{t}^{*}\left(u_{x}(\mathcal{X}(t, x))\right)$ is an obvious solution of SPDE (4.3). 


\subsection{Reverse Engineering Problem}

We consider the converse point of view of the marginal utility characterization given by Theorem 4.1. More precisely, by taking as input an initial condition $U(0,)=.u($.$) and some monotonic$ solution of $\operatorname{SDE}(\mu, \sigma)$ and $\operatorname{SDE}(\tilde{\mu}, \tilde{\sigma})$, we propose an explicit way to recover all consistent utility $U$ generating this wealth as optimal process. In the classical expected utility framework, this reverse engineering problem has been considered by He and Huang [10] (1992) in a complete market. Since the class of dynamic utilities is larger than the class of Markovian utilities considered in [10], our problem is easier to solve. In particular, we establish that the only assumption we need is the monotonicity of the wealth process with respect to the initial wealth, plus some integrability conditions.

In the following theorem, we try to introduce minimum assumptions used in the different problems. Either we assume the strong assumption that the $\operatorname{SDE}(\mu, \sigma)$ is regular enough to have a unique monotonic strong solution $X$ whose the inverse $\mathcal{X}$ is a semimartingale or we make the existence of $X$ in addition to the existence of a solution $\mathcal{X}$ to the $\operatorname{SPDE}\left(\widehat{L}_{t, x}^{\mu, \sigma},-\sigma \partial_{x}\right)$.

Theorem 4.3. Let $\kappa \in \mathcal{R}$ be a volatility vector and $\sigma_{t}(x)=x \kappa_{t}(x), \mu_{t}(x):=r_{t} x+\sigma_{t}(x) . \eta_{t}^{\mathcal{R}}$ the coefficients of the SDE defining an admissible wealth process. As previously, let $\widehat{L}_{t, x}^{\mu, \sigma}:=$ $\frac{1}{2} \partial_{x}\left(\left\|\sigma_{t}(x)\right\|^{2} \partial_{x}\right)-\mu_{t}(x) \partial_{x}$ be its adjoint operator.

Similarly, let $\nu \in \mathcal{R}^{\perp}$ be an orthogonal volatility vector, and $\left(\tilde{\mu}(t, y):=-y r_{t}, \tilde{\sigma}(t, y)=y\left(\nu_{t}(y)-\right.\right.$ $\left.\eta_{t}^{\mathcal{R}}\right)$ ) the coefficients of the SDE defining an admissible state price density process.

Assumptions a) Strong assumptions Assume the $\operatorname{SDE}(\mu, \sigma)$ in the class $\mathcal{S}^{3, \delta}(\delta \in(0,1])$, so that $S D E(\mu, \sigma)$ has a unique monotonic solution $\mathbf{X}$, whose the inverse $\mathcal{X}$ is solution of the $S P D E$

$$
d \mathcal{X}(t, x)=-\mathcal{X}_{x}(t, x) \sigma_{t}(x) \cdot d W_{t}+\widehat{L}_{t, x}(\mathcal{X}) d t
$$

b) or WEAK ASSUMPtions Assume only the $S D E(\mu, \sigma)$ in the class $\mathcal{S}^{1, \delta}(\delta \in(0,1])$, and the existence of a solution $\mathcal{X}$ of the $\operatorname{SPDE}\left(\widehat{L}_{t, x},-\sigma \partial_{x}\right)$

c) Assume the $\operatorname{SDE}(\tilde{\mu}, \tilde{\sigma})$ in the class $\mathcal{S}^{2, \delta}$ with monotonic solution $Y$.

MAIN RESUlT (i) For any initial utility function $u$, the stochastic random field $\mathbf{V}$ defined by $V(t, x)=Y_{t}\left(u_{x}(\mathcal{X}(t, x))\right)$, if it is integrable near to zero, is the derivative of a consistent stochastic utility $\mathbf{U}$, solution of the SPDE (4.3) with optimal wealth process $\mathbf{X}=\mathcal{X}^{-\mathbf{1}}$, solution of the $\operatorname{SDE}(\mu, \sigma)$.

(ii) The derivative of the convex conjugate $\widetilde{\mathbf{U}}$ of $\mathbf{U}$ is $\widetilde{\mathbf{U}}_{\mathbf{y}}:=-\mathbf{V}^{-\mathbf{1}}$ with $\widetilde{U}_{y}(t, y)=X_{t}(-$ $\left.\tilde{u}_{y}(\mathcal{Y}(t, y))\right)$ where $\mathcal{Y}$ denote the inverse flow of $\mathbf{Y}$. Moreover, if the $S D E(\tilde{\mu}, \tilde{\sigma})$ belongs to $\mathcal{S}^{3, \delta}$, the processes $\left(\widetilde{U}_{y}(t, y)\right)$ and $(\widetilde{U}(t, y))$ are Itô's semimartingales.

Proof. Calculations are easy consequence of Theorem 2.7 (ii), applied to the processes $\mathcal{X}$ solution of the SPDE (4.6), and $Y$ in place of $X$ with initial condition $u_{x}$.

(i) With the notations of Theorem 4.7 we have

$$
\begin{aligned}
d V(t, x) & =\tilde{\sigma}_{t}(V(t, x)) \cdot d W_{t}+\tilde{\mu}_{t}(V(t, x)) d t \\
& -\partial_{x} V(t, x) \sigma_{t}(x)\left[d W_{t}+\tilde{\sigma}_{t}(V(t, x)) / V(t, x) d t+\widehat{L}_{t, x}(V) d t\right.
\end{aligned}
$$

Recall that $\tilde{\sigma}_{t}(y)=y\left(\nu_{t}(y)-\eta_{t}^{\mathcal{R}}\right)$, so that $\tilde{\sigma}_{t}(y) \cdot \sigma_{t}(x)=-y \eta_{t}^{\mathcal{R}} \cdot \sigma_{t}(x)$. So, the process $V$ satisfies 
the SPDE (4.3). As in the proof of Theorem 4.1] the drift $\beta^{V}$ may be transformed into

$$
\begin{aligned}
\beta^{V}(t, x) & \left.=\tilde{\mu}_{t}(V(t, x))-\eta_{t}^{\mathcal{R}} \cdot \sigma_{t}(x) V_{x}(t, x)\right)+\partial_{x}\left(\frac{1}{2} V_{x}(t, x)\left\|\sigma_{t}(x)\right\|^{2}\right)-\mu_{t}(x) V_{x}(t, x) \\
& =-\left[r_{t} V(t, x)+V_{x}(t, x)\left(-x r_{t}+\eta_{t}^{\mathcal{R}} \cdot \sigma_{t}(x)-\eta_{t}^{\mathcal{R}} \cdot \sigma_{t}(x)\right)+\partial_{x}\left(\frac{1}{2} V_{x}(t, x)\left\|\sigma_{t}(x)\right\|^{2}\right)\right] \\
& =-\partial_{x}\left(V(t, x) x r_{t}\right)+\partial_{x}\left(\frac{1}{2} V_{x}(t, x)\|\sigma(t, x)\|^{2}\right)
\end{aligned}
$$

Similarly, the diffusion characteristic of $V, \gamma^{V}$ is given by

$$
\begin{aligned}
\gamma^{V}(t, x) & =\tilde{\sigma}_{t}(V(t, x))-V_{x}(t, x) \sigma_{t}(x) \\
& =V(t, x) \nu_{t}(V(t, x))-\eta_{t}^{\mathcal{R}} \cdot V(t, x)-V_{x}(t, x) \sigma_{t}(t, x)
\end{aligned}
$$

(ii) We recognize that $V$ has the same local characteristics that marginal of consistent utility (Theorem 4.1). Taking the primitive of $V$, (if that makes sense) we define a random field $U(t, x)=$ $\int_{0}^{x} V(t, z) d z=\int_{0}^{x} Y\left(t, u_{x}(\mathcal{X}(t, z))\right) d z$ which is a progressive utility satisfying the HJB constraint, $\beta^{U}(t, x)=-U_{x}(t, x) x r_{t}+\frac{1}{2} U_{x x}(t, x)\left\|\sigma_{t}(t, x)\right\|^{2}$ and the diffusion constraint $\gamma_{x}^{U}(t, x)=\gamma^{V}(t, x)=$ $U_{x}(t, x) \nu_{t}\left(U_{x}(t, x)\right)-\eta_{t}^{\mathcal{R}} . U_{x}(t, x)-U_{x x}(t, x) x \kappa(t, x)$. So, $U$ is a consistent dynamic utility.

Corollary 4.4. With the same notations as in Theorem 4.7, assume in addition global Lipschitz regularity on $(\mu, \sigma)$, that is $\sigma \in \tilde{\mathcal{K}}_{\mathbf{b}}^{0,1} \cap \tilde{\mathcal{K}}_{\text {loc }}^{3, \delta}, \nu \in \tilde{\mathcal{K}}_{\mathbf{b}}^{0,1} \cap \tilde{\mathcal{K}}_{\text {loc }}^{2, \delta}$ for $\delta \in(0,1]$. Let us also consider a utility function $u$ satisfying Inada's conditions, such that $u_{x} \sim x^{-\zeta}(\zeta<1)$ in the neighborhood of $z=0$.

The composite random field $Y_{t}\left(u_{x}(\mathcal{X}(t, x))\right)$ is integrable near to zero and it is the derivative of a consistent stochastic utility $U$.

Proof. Since $\sigma_{t}(x)=x \kappa_{t}(x) \in \tilde{\mathcal{K}}_{b}^{0,1} \cap \tilde{\mathcal{K}}_{l o c}^{3, \delta}, \nu_{t}(y) \in \tilde{\mathcal{K}}_{b}^{0,1} \cap \tilde{\mathcal{K}}_{l o c}^{2, \delta}$ for $\delta \in(0,1]$ one can easily shows that the pair $(\mu, \sigma)$ belong to $\left(\mathcal{K}_{b}^{0,1} \cap \mathcal{K}_{l o c}^{3, \delta}\right) \times\left(\tilde{\mathcal{K}}_{b}^{0,1} \cap \tilde{\mathcal{K}}_{l o c}^{3, \delta}\right)$ and $(\tilde{\mu}, \tilde{\sigma})$ to $\left(\mathcal{K}_{b}^{0,1} \cap \mathcal{K}_{l o c}^{2, \delta}\right) \times\left(\tilde{\mathcal{K}}_{b}^{0,1} \cap \tilde{\mathcal{K}}_{l o c}^{2, \delta}\right)$. Then, all assumptions of Theorem 4.7 are satisfied. Moreover, from Theorem 1.2, the unique strong monotonic solutions $X$ and $Y$ satisfy the following asymptotic behavior (equation (1.7))

$$
\lim _{z \rightarrow 0}\left(\sup _{0 \leq t \leq T} \frac{Z(t, z)}{z^{\varepsilon}}\right)=0 \text { and } \lim _{z \rightarrow 0}\left(\sup _{0 \leq t \leq T} \frac{Z(t, z)}{z^{1+\varepsilon}}\right)=+\infty, \text { for all } T
$$

Consequently, one can easily shows that, for any utility function $u$ satisfying Inada's conditions s.t. $u_{x}(x)<x^{-\zeta}$ for some $\zeta<1$, the composite random field $Y_{t}\left(u_{x}(\mathcal{X}(t, x))\right)$ is also integrable near to zero. Indeed, it suffices to write that for any $\alpha, \beta, \gamma>0$ we have

$$
\begin{aligned}
\lim _{x \rightarrow 0} x^{\alpha} Y_{t}\left(u_{x}(\mathcal{X}(t, x))\right) & =\lim _{x \rightarrow 0}\left(X_{t}(x)\right)^{\alpha} Y_{t}\left(u_{x}(x)\right)=\lim _{y \rightarrow+\infty}\left(X_{t}\left(-\tilde{u}_{y}(y)\right)\right)^{\alpha} Y_{t}(y) \\
& =\lim _{y \rightarrow+\infty}\left(\frac{X_{t}\left(-\tilde{u}_{y}(y)\right)}{\left(-\tilde{u}_{y}(y)\right)^{\beta}}\right)^{\alpha}\left(-\tilde{u}_{y}(y) y^{\gamma}\right)^{\alpha \beta} \frac{Y_{t}(y)}{y^{\alpha \beta \gamma}}
\end{aligned}
$$

As $u_{x}(x) \sim x^{-\zeta}$, we have $\lim _{x \rightarrow 0} x^{\gamma^{\prime}} u_{x}(x)=\lim _{y \rightarrow+\infty}-y^{\frac{1}{\gamma^{\prime}}} \tilde{u}_{y}(y)=0$ for any $\zeta<\gamma^{\prime}<1$. Taking $\gamma=\frac{1}{\gamma^{\prime}}>1, \varepsilon>0$ and $\beta=1+\varepsilon$ we deduce, from the asymptotic behavior of $X^{*}$ and $Y^{*}$ and Inada's conditions, that

$$
\lim _{x \rightarrow 0} x^{\alpha} Y_{t}\left(u_{x}(\mathcal{X}(t, x))\right)=\lim _{y \rightarrow+\infty}\left(\frac{X_{t}\left(-\tilde{u}_{y}(y)\right)}{\left(-\tilde{u}_{y}(y)\right)^{\beta}}\right)^{\alpha}\left(-\tilde{u}_{y}(y) y^{\gamma}\right)^{\alpha \beta} \frac{Y_{t}(y)}{y^{\alpha \beta \gamma}}=0, \forall \alpha \in\left(0, \frac{1}{\beta \gamma}\right) \subset(0,1),
$$

which shows the integrability near to zero of $Y_{t}\left(u_{x}(\mathcal{X}(t, x))\right)$. 
Risk tolerance dynamics With the utility characterization given in Theorem 4.7 the study of the risk tolerance coefficient, taken along the optimal wealth, is greatly simplified. In particular, the martingale property established in He and Huang in [10] in a complete market, $Y^{*}=y Y^{0}$ is easy to understand. A similar study has been performed by Zariphopoulou \& Zhou [39] in the special case of dynamic utility deduced by change of numeraire (see next section) and stochastic environment from a deterministic time depending utility function.

Proposition 4.5. Assume the optimal state density process to be the minimal one $Y^{*}(., y)=y Y_{\text {. }}^{0}$, or equivalently the orthogonal risk premium to be 0 . Let $U$ be a consistent dynamic utility with optimal regular wealth $X^{*}$ with derivative $X_{x}^{*}$.

(i) The path of the risk tolerance coefficient $\tau^{U}(t, x)=-\frac{U_{x}(t, x)}{U_{x x}(t, x)}$ at benchmark optimal wealth is given by $\tau^{U}\left(t, X_{t}^{*}(x)\right):=\tau^{*}(t, x)=\tau^{u}(x) X_{x}^{*}(t, x)$.

(ii) $\tau^{U}\left(t, X_{t}^{*}(x)\right)=\tau^{*}(t, x)$ is an admissible portfolio with initial wealth $\tau^{u}(x)$ and admissible allocation $\kappa_{t}^{d}=\kappa_{t}^{*}\left(X_{t}^{*}\right)+X_{t}^{*} \kappa_{x}^{*}\left(t, X_{t}^{*}\right)$. In particular, $Y_{t}^{0} \tau^{*}(t, x)$ is a local martingale.

(iii) As in [39], the pair $\left(X^{*}, \tau^{*}\right)$ is solution of two dimensional SDE with random coefficients.

Proof. (i) We start with the representation of the marginal utility as $\left.U_{x}(t, x)=Y_{t}^{0} u_{x}(\mathcal{X}(t, x))\right)$, and of its derivative $\left.U_{x x}(t, x)=Y_{t}^{0} u_{x x}(\mathcal{X}(t, x)) \partial_{x} \mathcal{X}(t, x)\right)=Y_{t}^{0} u_{x x}(\mathcal{X}(t, x)) / X_{x}^{*}(t, \mathcal{X}(t, x))$. By taking the ratio of these two quantities, we have that

$$
\tau^{U}(t, x)=\tau^{u}(\mathcal{X}(t, x)) X_{x}^{*}(t, \mathcal{X}(t, x)) \quad \text { or equivalently } \quad \tau^{U}\left(t, X_{t}^{*}(x)\right)=\tau^{u}(x) X_{x}^{*}(t, x)
$$

(ii) This last characterization is interesting since from Equation (2.4)

$$
d X_{x}^{*}(t, x)=X_{x}^{*}(t, x)\left[r_{t} d t+\sigma_{x}^{*}\left(t, X_{t}^{*}\right) \cdot\left(d W_{t}+\eta_{t}^{\mathcal{R}} d t\right)\right]
$$

As in the proof of Theorem 4.1 assertion $i)$, since $\mathcal{R}$ is a vector space, $X_{x}^{*}(t, x)$ is still an admissible portfolio with allocation policy $\kappa_{t}^{d}=\sigma_{x}^{*}\left(t, X_{t}^{*}\right)=\kappa_{t}^{*}\left(X_{t}^{*}\right)+X_{t}^{*} \kappa_{x}^{*}\left(t, X_{t}^{*}\right)$.

(iii) The pairs of processes $\left(X^{*}(t, x), X_{x}^{*}(t, x)\right)$, and $\left(X^{*}(t, x), \tau^{*}(t, x)\right)$ are solutions of the same 2-dimensional SDE.

All results of the proposition can be extended to the general case, where $Y_{t}^{*}(y)$ is no more a linear function of $y$, but the interpretation of the results is more difficult.

Proposition 4.6. Let $U$ be a $\mathcal{K}_{\text {loc }}^{2, \delta} \cap \mathcal{C}^{3}$-consistent dynamic utility $(\delta>0)$, then

(i) The risk tolerance coefficient $\tau^{U}$ is given by

$$
\tau^{U}(t, x)=\frac{Y_{t}^{*}\left(u_{x}(\mathcal{X})\right) X_{x}^{*}(t, \mathcal{X})}{u_{x x}(\mathcal{X}) Y_{x}^{*}\left(t, u_{x}(\mathcal{X})\right)}=\left(\tau^{u}(\mathcal{X}) \frac{Y_{t}^{*}\left(u_{x}(\mathcal{X}) X_{x}^{*}(t, \mathcal{X})\right.}{u_{x}(\mathcal{X}) Y_{y}^{*}\left(t, u_{x}(\mathcal{X})\right)}\right)(t, x)
$$

and, along the optimal wealth, $\tau^{U}\left(t, X_{t}^{*}(x)\right)=\frac{Y_{t}^{*}\left(u_{x}(x)\right)}{Y_{y}^{*}\left(t, u_{x}(x)\right) u_{x x}(x)} X_{x}^{*}(t, x)$.

(ii) The derivative of the optimal wealth is an admissible portfolio associated with the allocation $\kappa_{t}^{d}=\kappa_{t}^{*}\left(X_{t}^{*}\right)+X_{t}^{*} \kappa_{x}^{*}\left(t, X_{t}^{*}\right)$ and initial wealth 1 .

(iii) The process $Y_{y}^{*}(t, y) \tau^{U}\left(t, X_{t}^{*}(x)\right)$ is a local martingale.

The proof of this proposition is obvious. 
Pathwise dynamic programming principle In this paragraph we are interested in using the previous marginal utility characterization from a backward point of view. Unlike the standard case, we obtain a pathwise dynamic programming principle.

For this, observe that the identity $U_{x}(t, x)=Y_{t}^{*}\left(u_{x}(\mathcal{X}(t, x))\right)$ leads to the following identity based on the inverse flow $\mathcal{Y}$ of $Y^{*}, u_{x}(x)=\mathcal{Y}_{t}\left(U_{x}\left(t, X_{t}^{*}(x)\right)\right)$. This explains how to recover the marginal utility at time 0 from the stochastic terminal one at a time $t$. This point of view is interesting, because in this case the initial condition $u_{x}$ is simply the derivative of the value function of a classical optimization problem. To go further into this idea, properties of stochastic flows, previously not used (as explained above), will be essential.

\section{Flow property.}

(i) Let $\left(Z_{t}(x)\right)$ be a strictly monotonic random field with respect to $x$ with range $[0, \infty)$ whose the inverse random field is denoted by $\mathcal{Z}_{t}(z)$. We extend the random field $\mathbf{Z}$ to intermediate dates $(0 \leq s \leq t)$ by defining $Z_{t}(s, x)=Z_{t}\left(\mathcal{Z}_{s}(x)\right)$. The following classical notation $Z_{t}(s, x):=Z_{s, t}(x)$ is useful to express the semi-group property, that is for $s \leq t \leq u, Z_{t, t}(x)=x$ and $Z_{t, u} \circ Z_{s, t}=Z_{s, u}$. Note that $Z_{t}(x)=Z_{0, t}(x)$.

Similarly, we can extended the random field $\mathcal{Z}$ to intermediate dates by taking the inverse of $\left(Z_{t}(s, x)\right)$ such that $\mathcal{Z}_{t}(s, z)=Z_{s}\left(\mathcal{Z}_{t}(z)\right):=\mathcal{Z}_{s, t}(z)$. Then $\mathcal{Z}_{t}(s, z)=\mathcal{Z}_{s, t}(z)$ is a backward flow achieving the amount $z$ at date $s$. Then, the semi-group property holds true with the inverse order of the dates, that is for $s \leq t \leq u, \mathcal{Z}_{s, t} \circ \mathcal{Z}_{t, u}=Z_{s} \circ \mathcal{Z}_{t} \circ Z_{t} \circ \mathcal{Z}_{u}=\mathcal{Z}_{s, u}$.

(ii) When the flow $Z_{t}(x)$ is the monotonic solution of $\operatorname{some} \operatorname{SDE}(\mu, \sigma)$ starting from $x$ at time 0 , a classical result states that $Z_{s, t}(x)$ is a solution defined on $[s, \infty)$ of the same $\operatorname{SDE}(\mu, \sigma)$ starting from $x$ at time $s$. When the $\operatorname{SDE}(\mu, \sigma)$ is regular enough so that $\mathcal{Z}_{t}(z)$ is the monotonic solution of the $\operatorname{SPDE}(\mu, \sigma)$, then $\mathcal{Z}_{s, t}(z)$ is solution on $[s, \infty)$ of the same SPDE with initial condition $z$ at time $s$. Given that $\mathcal{Z}_{s, t}(z)=Z_{s}\left(\mathcal{Z}_{t}(z)\right)$, this result may be viewed as a consequence of Theorem 2.7 (i), applied to the regular function $G(y)=Z_{s}(y)$ considered as deterministic after the time $s$. But, it is more natural to consider $\mathcal{Z}_{s, t}(z)$ as a process in $s$ and so to introduce, time reversal and backward integration as in Kunita [19] Section 4.5, or Carmona \& Nualart [2]. We do not develop this point of view here.

Let us come back to consistent utility framework.

Proposition 4.7. We adopt the same framework as in Theorem with the monotonic solutions $X$ and $Y$ of two $S D E(\mu, \sigma)$ and $S D E(\tilde{\mu}, \tilde{\sigma})$. As usual the inverse processes are denoted by $\mathcal{X}$ and $\mathcal{Y}$. The marginal utility is defined as $U_{x}(t, x)=Y_{t}\left(u_{x}\left(\mathcal{X}_{t}(x)\right)\right)$.

(i) For any $t$, the pathwise identity holds true, $u_{x}(x)=\mathcal{Y}_{t}\left(U_{x}\left(t, X_{t}(x)\right)\right)$. This property is close to the following one, also true on the classical backward point of view,

$$
u_{x}(x)=\mathcal{Y}_{t}\left(U_{x}\left(t, X_{t}(x)\right)\right), \quad u_{x}(x)=\mathbb{E}\left(U_{x}(t, X(t, x)) X_{x}(t, x)\right)
$$

(ii) More generally, using the stochastic flows $X_{s, t}^{x}, Y_{s, t}^{y}, \mathcal{X}_{s, t}^{z}, \mathcal{Y}_{s, t}^{u}$, we have the pathwise dynamic principle

$$
U_{x}(t, x)=Y_{s, t}\left(U_{x}\left(s, \mathcal{X}_{s, t}(x)\right)\right), \quad U_{x}(s, x)=\mathcal{Y}_{s, t}\left(U_{x}\left(t, X_{s, t}(x)\right)\right),
$$

Proof. The both identities are easily deduced from the identity

$$
u_{x}\left(\mathcal{X}_{t}(x)\right)=\mathcal{Y}_{t}\left(U_{x}(t, x)\right)=u_{x}\left(\mathcal{X}_{s}\left(\mathcal{X}_{t, s}(x)\right)=\mathcal{Y}_{s}\left(U_{x}\left(s, \mathcal{X}_{t, s}(x)\right)\right) .\right.
$$


Comment The link between HJB SPDE and the inverse flows of some SDEs has received little attention in the literature to our knowledge, may be essentially because people are more interested by the Markovian case.

\section{Openness to other topics and works}

We close the paper by some openness to other topics and works; we show the stability of the notion of consistent utility by change of numeraire and then, without loss of generality, we can consider the martingale market where the portfolios are simple local martingales and the stochastic PDE's are easier to deal with. We also apply our method to the specific example of decreasing consistent utilities (see [1] and [28]) where the volatility vector $\gamma$ is given equal to zero, given a new interpretation of the optimal wealth as solution of inf-convolution problems in random power utilities.

\subsection{Change of numeraire}

Notations about the investment universe are the same as in Section 3 . A numeraire is a monetary reference used as payment instrument. When transactions take place in a given country, the domestic currency is used as numeraire, but in an international setting, a common numeraire is used in general for all the transactions. Because an investor is often faced with investing in different markets, we are concerned in this paragraph, by the impact of the change of numeraire on its progressive utility random field.

It is well-known (see Geman, El Karoui, and Rochet [9]) that the self-financing property is invariant by change of numeraire. So, if $N$ is an Itô positive continuous semimartingale, solution of linear equation with coefficients $\left(\mu^{N} x, \delta^{N} x\right)$, it is well-known (see also Platen and Heath, or Karatzas and Kardaras, [9, 31, 15]) that using $N$ as new numeraire transforms an Itô market with risk premium $\eta^{\mathcal{R}}$ and short rate $r_{t}$ into an investment universe, where the admissible portfolios are the processes $\widehat{X}^{\hat{\kappa}}=X^{\kappa} / N$ and the state price density processes are the processes $\widehat{Y}^{\nu}=N . Y^{\nu}$. The parameters of this new market are $\hat{\eta}=\eta^{\mathcal{R}}-\delta^{N}$, for the risk premium and $\hat{r}=r-\mu^{N}+\delta^{N} \cdot \eta^{\mathcal{R}}$ for the short rate.

(i) By the previous results, any $\mathcal{X}$-consistent dynamic utility $U$ defines a $\mathcal{X}^{N}$-consistent dynamic utility $U^{N}$ by the transformation $U^{N}(t, \hat{x})=U\left(t, \hat{x} N_{t}\right)$. Then the conjugate $\widetilde{U}^{N}$ is given by $\widetilde{U}^{N}(\hat{y})=\widetilde{U}\left(t, \hat{y} / N_{t}\right)$.

(ii) The class $\mathcal{X}^{N}$ of the admissible portfolios is characterized by the processes $\hat{\kappa}=\kappa-\delta^{N}$. The vector spaces $\left(\mathcal{R}_{t} ; t \geq 0\right)$ are transformed into affine spaces $\widehat{\mathcal{R}}_{t}=\mathcal{R}_{t}-\delta_{t}^{N}$. Nevertheless if $\delta_{t}^{N} \in \mathcal{R}_{t}$, for any time $t$, the constraint spaces $\widehat{\mathcal{R}}_{t}$ in the new market are the same than the constraint spaces $\mathcal{R}_{t}$ in the initial market.

(iii) The associated optimal portfolio is $X^{N, *}=X^{*} / N$ and the optimal state price density process is $Y^{N, *}=Y^{*} / N$.

(iv) The diffusion characteristic $\gamma^{N}(t, \hat{x})$ is obtained from Itô-Ventzel's formula, $\gamma^{N}(t, \hat{x})=\gamma\left(t, \hat{x} . N_{t}\right)+$ $\hat{x} U_{\hat{x}}^{N}(t, \hat{x}) \delta_{t}^{N}$. The new drift $\beta^{N}(t, \hat{x})$ is more complicated to described, and the explicit form is 
not of great interest in the general case.

(v) In the usual case, the market numeraire $\left(Y^{0}\right)^{-1}=M$ (also called numeraire portfolio, or growth optimal portfolio as in Platen and Heath [31]) is chosen as numeraire $Y^{0}$. Since $M$ is an admissible portfolio with volatility $\eta^{\mathcal{R}}$, the market parameters $\hat{\eta}$ and $\hat{r}$ are identically zero, and any admissible portfolio and any admissible state price density is a local martingale (under the historical probability).

The optimal policies are simply given by

$$
\hat{x} \hat{\kappa}_{t}^{*}(\hat{x})=\frac{\gamma_{\hat{x}}^{M, \mathcal{R}}(t, \hat{x})}{U_{\hat{x} \hat{x}}^{M}(t, \hat{x})}, \quad \hat{y} \hat{\nu}_{t}^{*}(\hat{y})=\frac{\tilde{\gamma}_{\hat{y}}^{M, \perp}(t, \hat{y})}{\widetilde{U}_{\hat{y} \hat{y}}^{M}(t, \hat{y})}
$$

while, the drift characteristics of the utility $U^{M}(t, \hat{x})$ and $\widetilde{U}^{M}(t, \hat{y})$ are given by,

$$
\beta^{M}(t, \hat{x})=-\frac{1}{2} U_{\hat{x} \hat{x}}^{M}(t, \hat{x})\left\|\hat{x} \kappa_{t}^{*, M}(\hat{x})\right\|^{2}, \tilde{\beta}^{M}(t, \hat{y})=-\frac{1}{2} \tilde{U}_{\hat{y} \hat{y}}^{M}(t, \hat{y})\left\|\hat{y} \nu_{t}^{*, N}(\hat{y})\right\|^{2},
$$

Consequently, $U^{M}$ is a supermartingale and $\widetilde{U}^{M}$ is a submartingale. Moreover, if $\gamma_{\hat{x}}^{M} \in \mathcal{R}$ the conjugate utility $\widetilde{U}^{M}$ is a local martingale and the optimal dual process is constant, $Y^{M, *} \equiv 1$. By symmetry, if $\gamma_{\hat{x}}^{M} \in \mathcal{R}^{\perp}, U^{M}(., \hat{x})$ is a local martingale for any $\hat{x}$, and the optimal wealth $X_{t}^{M, *}(\hat{x}) \equiv \hat{x}$.

\subsection{Decreasing Consistent Utilities}

In this section, all prices of the investment universe are assumed to be discounted, corresponding to the case where $r \equiv 0$. An interesting class of consistent utilities is the class of decreasing consistent utilities, which was studied and fully characterized in the literature by Musiela \& al. 28] and Berrier \& al. [1]. This utilities have a volatility characteristic $\gamma$ identically zero. It is an example where the dual SPDE is easier to study than the primal one, since by taking $\gamma=0$, it follows from Theorem 3.2 (3.6), that $U$ and $\widetilde{U}$ are solutions of the following SPDEs

$$
d U(t, x)=\frac{1}{2} \frac{U_{x}(t, x)^{2}}{U_{x x}(t, x)}\left\|\eta_{t}^{\mathcal{R}}\right\|^{2} d t, \quad d \widetilde{U}(t, y)=-\frac{1}{2} y^{2} \widetilde{U}_{y y}(t, y)\left\|\eta_{t}^{\mathcal{R}}\right\|^{2} d t
$$

which implies by convexity, that $t \mapsto U(t,),. \widetilde{U}(t, y)$ are decreasing functions.

Example of power utilities (i) It is easy to verify that the power dual utility functions $\widetilde{U}^{\vartheta}(t, y)=\frac{1}{1-\vartheta}\left(1-\tilde{C}_{t}^{\vartheta} y^{1-\vartheta}\right),(\vartheta>0)$, where as usual the parameter $\vartheta$ is the risk tolerance coefficient, are 5.1, if and only if $\tilde{C}_{t}^{\vartheta}(\omega)$ is solution of the ordinary equation $d \tilde{C}_{t}^{\vartheta}(\omega)=-\tilde{C}_{t}^{\vartheta}(\omega) \vartheta(1-$ $\vartheta)\left\|\eta_{t}^{\sigma}(\omega)\right\|^{2} d t$. In other words,

$\widetilde{U}^{\vartheta}(t, y)=\frac{1}{1-\vartheta}\left(1-\tilde{C}_{t}^{\vartheta} y^{1-\vartheta}\right), \tilde{C}_{t}^{\vartheta}=\exp \left(-\tilde{\epsilon}(\vartheta) A_{t}^{\eta}\right)$, with $A_{t}^{\eta}=\int_{0}^{t}\left\|\eta_{s}^{\mathcal{R}}\right\|^{2} d s, \tilde{\epsilon}(\vartheta)=(1-\vartheta) \vartheta>0$.

Then, $\widetilde{U}^{\vartheta}\left(t, y Y_{t}^{0}\right)-\frac{1}{1-\vartheta}=-\frac{y^{1-\vartheta}}{1-\vartheta}\left(Y_{t}^{0}\right)^{1-\vartheta} \tilde{C}_{t}^{\vartheta}$ is a martingale, since $y Y_{t}^{0}$ is the optimal state price density.

(ii) Let us observe that at any time $t>0$ the marginal conjugate utility $\widetilde{U}_{y}^{\vartheta}(t, y)=-\tilde{C}_{t}^{\vartheta} y^{-\vartheta}$ is no longer a monotonic function of the risk tolerance coefficient $\vartheta$ since the function $\vartheta \mapsto$ $\tilde{\epsilon}(\vartheta) A_{t}^{\eta}+\vartheta \ln (y)$ is no monotonic. 
(iii) The marginal power utility $U_{x}^{\vartheta}(t, x)$ is given by $U_{x}^{\vartheta}(t, x)=\left(\tilde{C}_{t}^{\vartheta}\right)^{1 / \vartheta} x^{-1 / \vartheta}$. Since $\left(Y_{t}^{0}\right)^{1-\vartheta} \tilde{C}_{t}^{\vartheta}$ is a local martingale, the process $X_{t}^{*, \vartheta}(x)=x \tilde{C}_{t}^{\vartheta}\left(Y_{t}^{0}\right)^{-\vartheta}$ is an admissible portfolio, and it is easy to see that it is the optimal one. Using the notation $\bar{X}_{t}^{\vartheta}=X_{t}^{*, \vartheta}(1)=\tilde{C}_{t}^{\vartheta}\left(Y_{t}^{0}\right)^{-\vartheta}$, we have $X_{t}^{*, \vartheta}(x)=x \bar{X}_{t}^{\vartheta}$.

(iv) As in the deterministic case, the optimal strategy $\kappa^{*, \vartheta}(t, x)$ is collinear to the risk premium $\eta_{t}^{\mathcal{R}}$ with factor $\vartheta, \kappa^{*, \vartheta}(t, x)=\vartheta \eta_{t}^{\mathcal{R}}$. Then, we recover the characterization of the marginal utility at the optimal state price density $y Y_{t}^{0}$ as $\widetilde{U}_{y}^{\vartheta}\left(t, y Y_{t}^{0}\right)=-\tilde{C}_{t}^{\vartheta} y^{-\vartheta}\left(Y_{t}^{0}\right)^{-\vartheta}=\tilde{u}_{y}^{\vartheta} \bar{X}_{t}^{\vartheta}$.

Characterization of decreasing conjugate utilities (i) The set of positive solutions $\widetilde{U}$ to the dual linear PDE [5.1] is a convex cone, stable by positive linear combination. From this, it is natural to consider the integral of conjugate power utilities, with respect to some positive Borel measure $m$, including the initial condition. The avoid the problem related to the constants, we formulate the problem on the marginal conjugate utilities and assume that $\int_{\mathbb{R}_{+}^{*}} y^{-\vartheta} d m(\vartheta)<\infty$, to define the new conjugate marginal utility

$$
\widetilde{U}_{y}^{m}(t, y)=\int_{\mathbb{R}_{+}^{*}} \widetilde{U}_{y}^{\vartheta}(t, y) \tilde{C}_{t}^{\vartheta} d m(\vartheta), \quad \tilde{u}_{y}^{m}(y)=-\int_{\mathbb{R}_{+}^{*}} y^{-\vartheta} d m(\vartheta)
$$

Such assumption on the initial condition $\tilde{u}_{y}(y)$ (then denoted $\tilde{u}_{y}^{m}(y)$ ) is equivalent to say that $\tilde{u}_{y}^{m}\left(e^{z}\right)$ is a completely monotonic function. All conjugate utility functions $\tilde{u}(y)$ do not verify this condition, but if it the case and if $m$ is compactly supported, it is easy to check that any primitive $\widetilde{U}^{m}(t, y)$ verifies the random PDE (5.1), since the property is true for the power conjuguate functions $\widetilde{U}_{y}^{\vartheta}(t, y)$ using Dirac measure at $\vartheta$ as shown in page 34 In other words, $\widetilde{U}^{m}(t, y)(\omega)$ is a space-time harmonic function of a geometrical Brownian motion with variance $A_{t}(\omega)$. By a generalization of Widder's Theorem [38], Musiela \& al. 228] and Berrier \& al. [1] showed that there is no other solution to the random PDE (5.1).

Sup-convolution interpretation (i) There is an interesting interpretation of these stochastic utilities: one can imagine an investor starting with power utility, with some ambiguity on its risk tolerance coefficient. At time 0 , she starts with a mixture of marginal conjugate power utilities, weighted by some measure $m$. At time $t$, the marginal conjugate consistent utility is still a mixture of power conjugate utilities with respect to the measure $m_{t}(d \vartheta):=\tilde{C}_{t}^{\vartheta} d m(\vartheta)$, with decreasing in time random density $\tilde{C}_{t}^{\vartheta}$. The stochastic measure $m_{t}(d \vartheta)$ is the unique measure which ensure that the process $\widetilde{U}_{y}^{m}$ constructed in equation (5.2) is the derivative of the conjugate of a consistent utility.

(ii) Optimal Wealth The characterization of the marginal conjugate utility $\widetilde{U}_{y}^{m}\left(t, y Y_{t}^{0}\right)$ along the optimal density process $y Y_{t}^{0}$ as $\widetilde{U}_{y}^{m}\left(t, y Y_{t}^{0}\right)=X_{t}^{*, m}\left(-\tilde{u}_{y}^{m}(y)\right)$ is useful to characterize the optimal wealth $X_{t}^{*, m}(x)$, since the same property is true for the standard power utility functions,

$$
X_{t}^{*, m}\left(-\tilde{u}_{y}^{m}(y)\right)=\int_{\mathbb{R}_{+}^{*}} \widetilde{U}_{y}^{\vartheta}\left(t, y Y_{t}^{0}\right) d m_{t}(\vartheta)=\int_{\mathbb{R}_{+}^{*}} X_{t}^{*, \vartheta}\left(-\tilde{u}_{y}^{\vartheta}(y)\right) d m_{t}(\vartheta)=\int_{\mathbb{R}_{+}^{*}} \bar{X}_{t}^{\vartheta} y^{-\vartheta} d m_{t}(\vartheta),
$$

To give a decomposition directly in terms of $x$, we start with a family of well-chosen initial wealths $x^{\vartheta, m}$ satisfying $x^{\vartheta, m}(x)=-\tilde{u}_{y}^{\vartheta}\left(u_{x}^{m}(x)\right)$ so that $\left.\int_{\mathbb{R}^{*}} x^{\vartheta, m}(x)\right) d m(\vartheta)=-\tilde{u}_{y}^{m}\left(u_{x}^{m}(x)\right)=x$. So, the optimal wealth process $X_{t}^{*, m}$ issued from $x=-\tilde{u}_{y}^{m}\left(u_{x}^{m}(x)\right)$ is given by the closed formula

$$
X_{t}^{*, m}(x)=\int_{0}^{\infty} X_{t}^{*, \vartheta}\left(x^{\vartheta}(x)\right) m_{t}(d \vartheta)=\int_{0}^{\infty} x^{\vartheta}(x) \bar{X}_{t}^{\vartheta} m_{t}(d \vartheta), \text { with } \int_{0}^{\infty} x^{\vartheta}(x) m(d \vartheta)=x .
$$


$X_{t}^{*, m}(x)$ is strictly increasing and regular with respect to its initial condition $x$, since any function $x^{\vartheta, m}(x)$ is monotonic and regular. The conjuguate of $\widetilde{U}_{t}^{m}(y), U^{m}(t, x)$ is a consistent utility with optimal wealth $X_{t}^{*, m}(x)$.

(iii) The RISK TOLERANCE COEFFICIENT We are concerned with the risk tolerance coefficient $\tau_{U}^{m}(t, x)=-\frac{U_{x}^{m}(x)}{U_{x x}^{m}(x)}$ of the utility function $U^{m}(t, x)$, given that for power utility $\tau^{\vartheta}(t, x)=\vartheta x$.

According to assertion $(i)$ of Proposition 4.5 stating that $\tau_{U}^{m}\left(t, X_{t}^{*, m}(x)\right)=\tau_{u}^{m}(x) X_{x}^{*, m}(t, x)$, we see that

$$
\tau_{U}^{m}\left(t, X_{t}^{*, m}(x)\right)=\tau_{u}^{m}(x) X_{x}^{*, m}(t, x)=\int_{0}^{\infty} \tau_{u}^{m}(x) x_{x}^{\vartheta}(x) X_{x}^{*, \vartheta}\left(t, x^{\vartheta}(x)\right) m_{t}(d \vartheta)
$$

On the other hand, by the fact that $x_{x}^{\vartheta}(x)=-u_{x x}^{m}(x) \tilde{u}_{y y}^{\vartheta}\left(u_{x}^{m}(x)\right)$, we easily get the equality $\tau^{\vartheta}\left(x^{\vartheta}(x)\right)=x_{x}^{\vartheta}(x) \tau^{U}(x)$, and so and hence,

$$
\tau_{U}^{m}\left(t, X_{t}^{*, m}(x)\right)=\int_{0}^{\infty} \tau^{\vartheta}\left(t, X_{t}^{*, \vartheta}\left(x^{\vartheta}(x)\right)\right) m_{t}(d \vartheta)
$$

We still have some mixture properties along the optimal processes.

(iv) Sup-convolution interpretation Classical result in convex analysis shows the link between mixture of conjuguate utility functions and inf-convolution problem, in the following form: assume that the derivative of some conjuguate utility function $\tilde{u}_{y}^{m}(y)$ may be represented as the integral of some family of marginal conjuguate utility functions $\tilde{u}_{y}^{\vartheta}(y)$, with respect to some positive Borel measure $m, \tilde{u}_{y}^{m}(y)=\int_{0}^{\infty} \tilde{u}_{y}^{\vartheta}(y) m(d \vartheta)$, where the integral is finite for any $y$. Then, $\tilde{u}^{m}$ is the convex conjuguate of the sup-convolution problem associated with

$$
u^{m}(x)=\sup \left\{\int_{0}^{\infty} u^{\vartheta}\left(x_{\vartheta}\right) m(d \vartheta) \mid\left(x_{\vartheta}\right) \text { such that } \int_{0}^{\infty} x_{\vartheta} m(d \vartheta)=x\right\}
$$

The optimal solution $\left(x_{\vartheta}^{*}\right)$ that achieves the maximum in the optimization problem 5.5 is given explicitly as $x^{\vartheta, m}(x)=\tilde{u}_{y}^{\vartheta}\left(-u_{x}^{m}(x)\right)$.

Come back to our problem of decreasing consistent utility random field. The strategy associated with the initial condition $x^{\vartheta, m}(x)$ may be interpreted as the deterministic optimal allocations of the initial wealth with respect to the parameter $\vartheta$, in a Pareto optimal equilibrium where a continuum of agents with different risk aversion are in competition. The same interpretation holds true at time $t$, with the family of wealth $X_{t}^{*, \vartheta}\left(x^{\vartheta}(x)\right)$ and the random measure $m_{t}(d \vartheta)$.

Moreover, the decreasing consistent utility random field is given at any time $t$ as,

$$
U^{m}(t, x)=\sup \left\{\int_{0}^{\infty} U^{\vartheta}\left(t, X_{\vartheta}(t, x)\right) m_{t}(d \vartheta) \mid\left(X_{\vartheta}(t, x)\right) \text { such that } \int_{0}^{\infty} X_{\vartheta}(t, x) m_{t}(d \vartheta)=x\right\}
$$

(v)THE ROLE OF THE INITIAL UTILITY FUNCTION In the study of decreasing utilities, we introduced an assumption on the initial condition, that is $\tilde{u}_{y}^{m}(y)=-\int_{\mathbb{R}_{+}^{*}} y^{-\vartheta} d m(\vartheta)$. The Borel measure $m$ is determining in the definition of the optimal wealth. This may seem at odds with the rest of the paper. This is not the case and even this is a nice example to illustrate our results.

Indeed, starting from the optimal portfolio $X^{*, m}$ defining in Equation (5.3), it is easy to construct a new consistent utility (more easily its Fenchel conjuguate $\tilde{V}(t, y))$ having $\left(X^{*, m}, y Y^{0}\right)$ as optimal processes, starting from an initial concave function $v$ with Fenchel conjuguate $\tilde{v}$. Thanks 
to the dual characterization $\widetilde{V}_{y}^{m}\left(t, y Y_{t}^{0}\right)=X_{t}^{*, m}\left(-\tilde{v}_{y}(y)\right)$ of the marginal conjuguate utility, the same identity applied to $\widetilde{U}_{y}^{m}$ yields to the following relation $\widetilde{V}_{y}^{m}\left(t, y Y_{t}^{0}\right)=X_{t}^{*, m}\left(-\tilde{v}_{y}(y)\right)=$ $\widetilde{U}_{y}^{m}\left(t, u_{x}^{m}\left(-\tilde{v}_{y}(y)\right) Y_{t}^{0}\right)$. It remains to make the change of variable $y \mapsto y / Y_{t}^{0}$, to obtain the marginal dual conjuguate

$$
\widetilde{V}_{y}^{m}(t, y)=X_{t}^{*, m}\left(-\tilde{v}_{y}\left(y / Y_{t}^{0}\right)\right)=\widetilde{U}_{y}^{m}\left(t, u_{x}^{m}\left(-\tilde{v}_{y}\left(y / Y_{t}^{0}\right)\right) Y_{t}^{0}\right) .
$$

Obviously, by this transformation we lose the decreasing property in time of $\widetilde{V}_{y}^{m}(t, y)$ since $u_{x}^{m}\left(-\tilde{v}_{y}\left(y / Y_{t}^{0}\right)\right) Y_{t}^{0}$ is no more a decreasing process. The same kind of construction may be made when the optimal dual process $y Y_{t}^{0}$ is replaced by a monotonic one.

Note, similar ideas are developed in [5] to build richer classes of utilities.

Conclusion In this new approach, the solution of the utility SPDE have a pathwise representation, unlike to the characteristics method where the solutions are represented as a conditional expectation. There are several advantages of this connection between SPDEs and SDEs due to the many results of the SDE theory. To the best of our knowledge, there are no or few results that assert the monotonicity or the convexity of such solutions. Also, there may be other advantages in numerical methods and simulations of the SDE than of SPDE.

Otherwise, this paper investigates consistent stochastic utilities from the SPDE point of view. This leads therefore to make strong regularity assumptions: the market is a Brownian market and securities are modeled as continuous semimartingales. Utilities are at least of class $\mathcal{K}^{2}$ in the sense of Kunita in order to apply Itô-Ventzel's formula and to deduce the SPDEs. Moreover, the method of stochastic utilities construction is based on the dynamics of stochastic flows and their inverses, and therefore additional regularity assumptions on $X^{*}$ and $Y^{*}$ are required. However, one can take a direct approach still based on monotonicity assumptions on optimal processes for the primal and dual problem, and on compound flows formula ; it is showed in [6], that these assumptions can be considerably weakened. Indeed, considering any financial market in which the securities are modeled as bounded semimartingales, the stochastic utilities are of class $\mathcal{K}^{1}$ and wealth process are required to lie in a convex class $\mathscr{X} \subset \mathbb{X}^{+}$, the monotonicity assumption of $X^{*}$ and $Y^{*}$ is sufficient to show the validity of the construction proposed in this work, using analysis methods and optimality conditions.

\section{References}

[1] F. Berrier, C. Rogers, and M. Tehranchi. A characterization of forward utility functions. Preprint, 2009.

[2] R.A. Carmona and D. Nualart. Nonlinear stochastic integrators, equations and flows, volume 6 of Stochastics Monographs. Gordon and Breach Science Publishers, New York, 1990.

[3] P. Constantin and G. Iyer. Stochastic lagrangian transport and generalized relative entropies. Commun.Math.Sci, 4:767-777, 2006.

[4] D. Duffie and L. Epstein. Stochastic differential utility. Econometrica, 60(2):353-394, 1992. With an appendix by the authors and C. Skiadas. 
[5] N. El Karoui and M. Mrad. Mixture of consistent stochastic utilities, and a priori randomness. Preprint., 2010.

[6] N. El Karoui and M. Mrad. Stochastic utilities with a given optimal portfolio : approach by stochastic flows. Preprint., 2010.

[7] N. El Karoui, S. Peng, and M.C. Quenez. A dynamic maximum principle for the optimization of recursive utilities under constraints. Ann. Appl. Probab., 11(3):664-693, 2001.

[8] M. Frittelli and M. Maggis. Conditional certainty equivalent. International Journal of Theoretical and Applied Finance (IJTAF), 14(01):41-59, 2011.

[9] H. Geman, N. El Karoui, and J.C. Rochet. Change of numeraire, change of probability measure and option pricing. Journal of Applied Probability, 32:443-458, 1995.

[10] H. He and C. Huang. Consumption-portfolio policies: An inverse optimal problem. Journal of Economic Theory, 62(2):257 - 293, 1994.

[11] V. Henderson and D. Hobson. Horizon-unbiased utility functions. Stochastic Process. Appl., 117(11):1621-1641, 2007.

[12] P. Imkeller, S. Fang, and T. Zhang. Global flows for stochastic differential equations without global Lipschitz conditions. Ann. Probab., 35(1):180-205, 2007.

[13] P. Imkeller and M.K. Scheutzow. On the spatial asymptotic behavior of stochastic flows in Euclidean space. Ann. Probab., 27:109-129, 1999.

[14] I. Karatzas and N. Englezos. Utility maximization with habit formation: Dynamic programming and stochastic pdes. SIAM J. Control Optim., 48(2):481-520, 2009.

[15] I. Karatzas and C. Kardaras. The numéraire portfolio in semimartingale financial models. Finance Stoch., 11(4):447-493, 2007.

[16] I. Karatzas, J.P. Lehoczky, S.E. Shreve, and G.L. Xu. Martingale and duality methods for utility maximization in an incomplete market. SIAM J. Control Optim., 29(3):702-730, 1991.

[17] I. Karatzas and S.E. Shreve. Methods of Mathematical Finance. Springer, September 2001.

[18] D. Kramkov and W. Schachermayer. The asymptotic elasticity of utility functions and optimal investment in incomplete markets. Annals of Applied Probability, 9:904-950, 1999.

[19] H. Kunita. Stochastic flows and stochastic differential equations, volume 24 of Cambridge Studies in Advanced Mathematics. Cambridge University Press, 1997.

[20] Z. Liang. Stochastic differential equations driven by spatial parameters semimartingale with non-lipschitz local characteristic. Potential Analysis, 26:307-322, 2007.

[21] Z. Liang. Spatial asymptotic behavior of homeomorphic global flows for non-Lipschitz SDEs. Bull. Sci. Math., 132:146-163, 2008. 
[22] M. Mania and R. Tevzadze. Backward stochastic PDEs related to the utility maximization problem. Georgian Mathematical Journal, 17:705-740, 2010.

[23] S.E.A. Mohammed and M.K. Scheutzow. Spatial estimates for stochastic flows in Euclidean space. Ann. Probab., 26:56-77, 1998.

[24] M. Musiela and T. Zariphopoulou. Backward and forward utilities and the associated pricing systems: The case study of the binomial model. pages 3-44. Princeton University Press, 2005-2009.

[25] M. Musiela and T. Zariphopoulou. Investment and valuation under backward and forward dynamic exponential utilities in a stochastic factor model. In Advances in mathematical finance, pages 303-334. Birkhäuser Boston, 2007.

[26] M. Musiela and T. Zariphopoulou. Optimal asset allocation under forward exponential performance criteria. volume 4, pages 285-300. Institute of Mathematical Statistics, 2008.

[27] M. Musiela and T. Zariphopoulou. Portfolio choice under dynamic investment performance criteria. Quantitative Finance, 9(2):161-170, 2009.

[28] M. Musiela and T. Zariphopoulou. Portfolio choice under space-time monotone performance criteria. SIAM J. Financial Math., 1:326-365, 2010.

[29] M. Musiela and T. Zariphopoulou. Stochastic partial differential equations in portfolio choice. In Contemporary Quantitative Finance, pages 195-3216, 2010.

[30] H. Pham. Optimisation et contrôle stochastique appliqués à la finance, volume 61 of Mathématiques 83 Applications (Berlin). Springer, Berlin, 2007.

[31] E. Platen and D. Heath. A benchmark approach to quantitative finance. Springer Finance. Springer-Verlag, Berlin, 2006.

[32] P. Protter. Stochastic integration and differential equations, volume 21 of Stochastic Modelling and Applied Probability. Springer-Verlag, Berlin, 2005.

[33] R. Rouge and N.El Karoui. Pricing via utility maximization and entropy. Math. Finance, 10(2):259-276, 2000. INFORMS Applied Probability Conference (Ulm, 1999).

[34] W. Schachermayer. Optimal investment in incomplete financial markets. In Mathematical Finance: Bachelier Congress 2000, pages 427-462. Springer, 2001.

[35] M.K. Scheutzow. Chaining techniques and their application to stochastic flows. In London Mathematical Society Lecture Note Series (No. 353), pages 35-64. Cambridge University Press, 2009.

[36] C. Stricker, T. Choulli, and J. Li. Minimal Hellinger martingale measures of order $q$. Finance Stoch., 11(3):399-427, 2007. 
[37] A.D. Ventzel. On equations of the theory of conditional Markov. Theory of Probability and its Applications, 10:357-361, 1965.

[38] D. Widder. The heat equation. Academic Press Inc., U.S., 1976.

[39] T. Zariphopoulou and T. Zhou. Investment performance measurement under asymptotically linear local risk tolerance. In Handbook of numerical analysis. Vol XV, pages 227-253. Elsevier/North-Holland, 2009.

[40] X. Zhang. Stochastic flows of sdes with irregular coefficients and stochastic transport equations. Bulletin des Sciences MathÃAC)matiques, 134(4):340 - 378, 2010.

[41] G. Zitkovic. A dual characterization of self-generation and log-affine forward performances. Preprint, 2008. 